\title{
ActRIIB:ALK4-Fc alleviates muscle dysfunction and comorbidities in murine models of neuromuscular disorders
}

\author{
Jia Li, ${ }^{1}$ Maureen Fredericks, ${ }^{1}$ Marishka Cannell, ${ }^{1}$ Kathryn Wang, ${ }^{1}$ Dianne Sako, ${ }^{1}$ Michelle C. Maguire, ${ }^{1}$ Rosa Grenha, ${ }^{1}$ Katia Liharska, \\ Lavanya Krishnan, ${ }^{1}$ Troy Bloom, ${ }^{1}$ Elitza P. Belcheva, ${ }^{1}$ Pedro A. Martinez, ${ }^{1}$ Roselyne Castonguay, ${ }^{1}$ Sarah Keates, ${ }^{1}$ Mark J. Alexander, ${ }^{1}$ \\ Hyunwoo Choi, ${ }^{2}$ Asya V. Grinberg, ${ }^{1}$ R. Scott Pearsall, ${ }^{1}$ Paul Oh, ${ }^{2}$ Ravindra Kumar, ${ }^{1}$ and Rajasekhar N.V.S. Suragani ${ }^{1}$
}

'Acceleron Pharma Inc., Cambridge, Massachusetts, USA. ²Department of Neurobiology, Barrow Neurological Institute, Phoenix, Arizona, USA.

\begin{abstract}
Patients with neuromuscular disorders suffer from a lack of treatment options for skeletal muscle weakness and disease comorbidities. Here, we introduce as a potential therapeutic agent a heterodimeric ligand-trapping fusion protein, ActRIIB:ALK4-Fc, which comprises extracellular domains of activin-like kinase 4 (ALK4) and activin receptor type IIB (ActRIIB), a naturally occurring pair of type I and II receptors belonging to the TCF- $\beta$ superfamily. By surface plasmon resonance (SPR), ActRIIB:ALK4-Fc exhibited a ligand binding profile distinctly different from that of its homodimeric variant ActRIIB-Fc, sequestering ActRIIB ligands known to inhibit muscle growth but not trapping the vascular regulatory ligand bone morphogenetic protein 9 (BMP9). ActRIIB:ALK4-Fc and ActRIIB-Fc administered to mice exerted differential effects concordant with SPR results - on vessel outgrowth in a retinal explant assay. ActRIIB:ALK4-Fc induced a systemic increase in muscle mass and function in wild-type mice and in murine models of Duchenne muscular dystrophy (DMD), amyotrophic lateral sclerosis (ALS), and disuse atrophy. Importantly, ActRIIB:ALK4-Fc improved neuromuscular junction abnormalities in murine models of DMD and presymptomatic ALS and alleviated acute muscle fibrosis in a DMD model. Furthermore, in combination therapy ActRIIB:ALK4-Fc increased the efficacy of antisense oligonucleotide M12-PMO on dystrophin expression and skeletal muscle endurance in an aged DMD model. ActRIIB:ALK4-Fc shows promise as a therapeutic agent, alone or in combination with dystrophin rescue therapy, to alleviate muscle weakness and comorbidities of neuromuscular disorders.
\end{abstract}

\section{Introduction}

Neuromuscular disorders (NMDs) encompass a wide range of conditions with impaired muscle function. Such disorders include disuse atrophy, in which unloading and inactivity cause loss of muscle mass (1); sarcopenia, which occurs as a result of the aging process; and cachexia, in which muscle wasting takes place due to a chronic illness such as cancer (2). NMDs can also be caused by either inherited or spontaneous genetic mutations (3). These mutations can give rise to myopathies that directly affect the voluntary muscle, as in the case of the muscular dystrophies, or they can cause progressive degeneration of the motor neurons that leads to muscle weakness, as in the case of amyotrophic lateral sclerosis (ALS) and Charcot-Marie-Tooth disease (3). The outcome for many patients with NMDs is a profound deterioration in quality of life and a significant increase in the burden of care (4).

Conflict of interest: PO has received compensation from Acceleron Pharma. JL, MF, MC, KW, DS, MCM, RG, KL, LK, TB, EPB, PAM, RC, SK, MJA, AVG, RSP, RK, and RNVSS either are or have been employees of Acceleron Pharma and either own or have owned stock in Acceleron Pharma.

Copyright: (ㄷ 2021, American Society for Clinical Investigation.

Submitted: April 6, 2020; Accepted: December 29, 2020; Published: February 15, 2021

Reference information: J Clin Invest. 2021;131(4):e138634.

https://doi.org/10.1172/JCl138634
Duchenne muscular dystrophy (DMD) is an X-linked myopathy caused by loss-of-function mutations in the dystrophin gene (DMD) (5), which encodes a cytoskeletal protein that helps to connect the internal cytoskeleton to the extracellular matrix (6). Dystrophin deficiency weakens membrane integrity, leading to myofiber damage during muscle contraction, and eventually muscle is replaced by fat and connective tissue (7). With disease progression the muscles of the cardiac and respiratory system are also affected, and DMD patients die prematurely due to respiratory failure or cardiomyopathy (8). There is presently no cure for DMD, and patients are typically managed using supportive measures and through the use of corticosteroids (9), although therapies to restore dystrophin expression via either exon skipping or micro-dystrophin gene replacement are under development (10). One of these therapies, Exondys51, has been recently approved by the FDA and could potentially benefit more than $10 \%$ of the DMD population $(11,12)$. However, this treatment increases dystrophin expression in the skeletal muscle to less than $1 \%$ of normal levels and, importantly, has not yet demonstrated clinical and functional benefit to patients (13). Thus, there is still a high unmet therapeutic need to remedy muscle weakness and the comorbidities associated with DMD and other neuromuscular diseases.

The transforming growth factor- $\beta$ (TGF- $\beta$ ) superfamily signaling pathway plays an integral role in maintaining skeletal muscle homeostasis (14). When deficiency in, and inhibition of, growth 
A

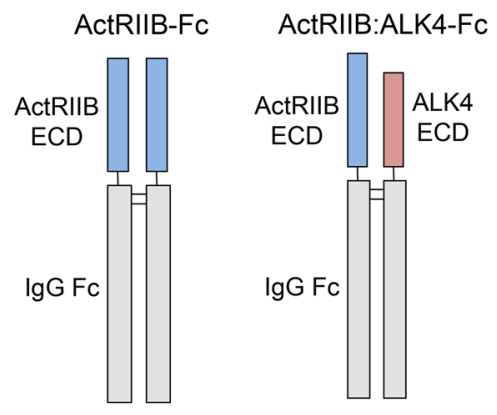

B

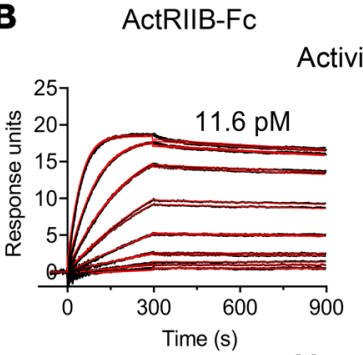

Myostatin
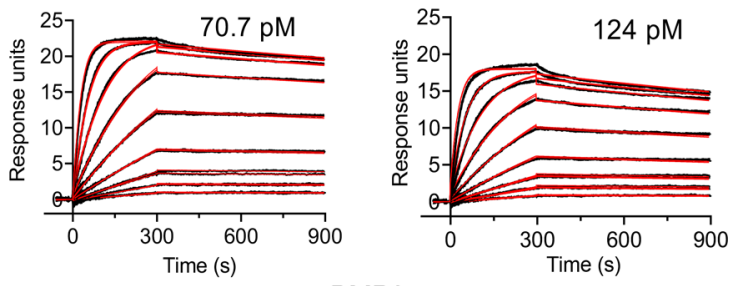

BMP9
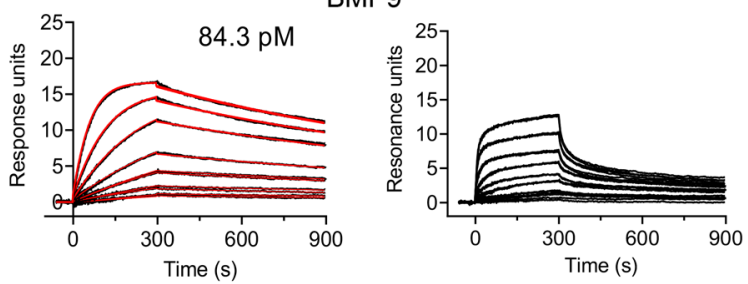

C

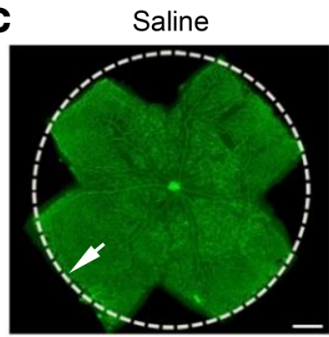

ActRIIB-Fc
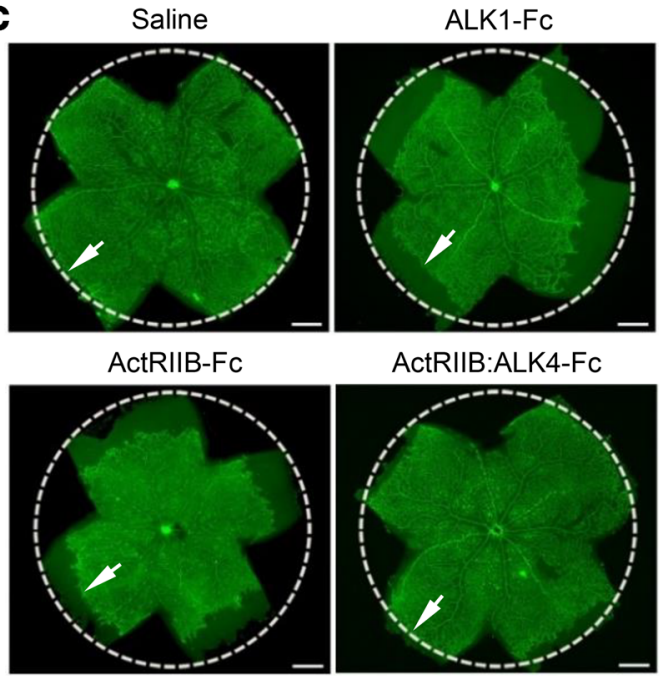

ActRIIB:ALK4-Fc

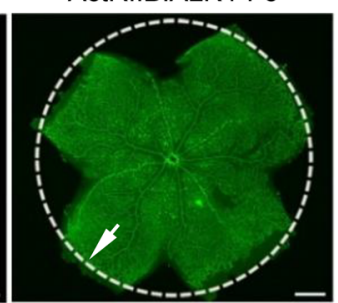

D

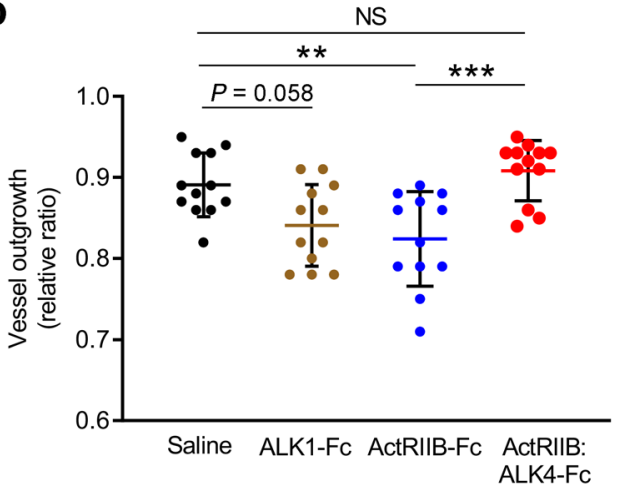

Figure 1. Heterodimeric fusion protein ActRIIB:ALK4-Fc does not inhibit BMP9. (A) Domain structure of ActRIIB:ALK4-Fc compared with homodimeric ActRIIB-Fc. (B) SPR sensorgrams showing binding of activin A, myostatin (GDF8), and BMP9 to ActRIIB-Fc and ActRIIB:ALK4-Fc. Sensorgrams (black lines) are overlaid with fits to a 1:1 interaction model with mass transport limitations (red lines). Kinetic parameters from these experiments are presented in Table 1. (C and D) C57BL/6 mice were treated on postnatal day 2 (P2) with saline, ALK1-Fc (positive control), ActRIIB-Fc, or ActRIIB:ALK4-Fc (2 mg/kg each), and retinas were prepared on P8. (C) Vessel staining of retinal flat mounts with IB4. White arrows indicate the retinal vascular front, and dashed circles represent extent of control vascular outgrowth. Scale bars: $500 \mu \mathrm{m}$. (D) Quantification of vascular outgrowth toward the retinal periphery, measured as the distance from the optic nerve to the outermost vessel in the vascular front. Each symbol corresponds to a separate retina and represents the average of 4 measurements per retina. Bars represent means \pm SEM ( $n=12$ per group). Group differences were assessed by 1-way ANOVA followed by Tukey's post hoc test. ${ }^{* *} P<0.01,{ }^{* *} P<0.001$.

differentiation factor 8 (GDF8; also known as myostatin, MSTN) were discovered to produce a muscular hypertrophy phenotype $(15,16)$, it expanded the possibilities for the treatment of NMDs. However, targeting of GDF8 alone, even with promising results at the preclinical stage, has not yet translated successfully in the clinical setting (17). Greater efficacy might be obtained by inhibition of multiple superfamily ligands, such as activin A, activin B, and GDF11, which act in concert with GDF8 to reduce muscle mass $(18,19)$. These ligands can signal through activin receptor type IIB (ActRIIB), which pairs with the type I receptors activin-like kinase 4 (ALK4) or ALK5 to activate canonical Smad2/3 signaling and suppress muscle growth (20). The homodimeric fusion protein ActRIIB-Fc was previously shown to sequester TGF- $\beta$ superfamily ligands to reduce Smad $2 / 3$ activation and increase muscle mass (21). However, ActRIIB-Fc also binds bone morphogenetic protein 9 (BMP9), which activates the Smad1/5/8 pathway, causing unwanted vascular effects in vivo (22).

The present study introduces a novel heterodimeric Fc fusion protein, ActRIIB:ALK4-Fc, which incorporates the extracellular domains of ActRIIB and ALK4 and can sequester Smad2/3 pathway ligands with novel selectivity. Our results demonstrate that ActRIIB:ALK4-Fc robustly improves muscle mass and function in wild-type (WT) mice and in multiple models of NMDs but, unlike the homodimeric ActRIIB-Fc, does not sequester BMP9 and thereby circumvents deleterious vascular effects associated with BMP9 inhibition. Importantly, we show that ActRIIB:ALK4-Fc treatment 


\section{Table 1. Ligand binding parameters for ActRIIB-Fc and ActRIIB:ALK4-Fc determined by SPR}

Ligand

ActRIIB-Fc

$\begin{array}{cc}k_{a} & k_{d} \\ \left(\times 10^{6} \mathrm{M}^{-1} \mathrm{~s}^{-1}\right) & \left(\times 10^{-6} \mathrm{~s}^{-1}\right)\end{array}$

$$
K_{D}
$$

$(\mathrm{pM})$

$\begin{array}{cc} & \text { ActRIIB:ALK4-Fc } \\ k_{a} & k_{d}\end{array}$

$K_{0}$

$\begin{array}{lcccccc}\text { Activin A } & 11.2 \pm 0.6 & 102.1 \pm 11.7 & 9.2 \pm 1.2 & 8.4 \pm 0.3 & 113.9 \pm 21.0 & 13.7 \pm 2.8 \\ \text { Activin B } & 12.2 \pm 0.2 & 62.2 \pm 6.9 & 5.1 \pm 0.6 & 10.6 \pm 0.5 & 84.3 \pm 10.7 & 8.1 \pm 1.3 \\ \text { CDF8 } & 2.6 \pm 0.2 & 136.5 \pm 9.1 & 53.4 \pm 5.8 & 2.0 \pm 0.1 & 326.6 \pm 31.7 & 162.6 \pm 12.0 \\ \text { CDF11 } & 6.2 \pm 0.6 & 206.3 \pm 19.3 & 33.4 \pm 0.4 & 4.2 \pm 0.7 & 198.0 \pm 10.8 & 55.2 \pm 12.0 \\ \text { BMP9 } & 5.9 \pm 0.7 & 660.5 \pm 18.9 & 116.1 \pm 10.8 & & \text { Transient }{ }^{A} & \end{array}$

Kinetic characterization and comparison of ligand binding to ActRIIB-Fc and ActRIIB:ALK4-Fc as assessed by SPR. Data were globally fit to a 1:1 binding model with mass transfer using BIAevaluation Software. Data are means \pm SEM ( $n=4-5$ replicates). ${ }^{A}$ Transient binding characterized by rapid association and dissociation of the complex, which prevents accurate $k_{a}$ and $k_{d}$ determination.

in DMD mouse models alleviates comorbidities of DMD such as fibrosis and abnormalities of the neuromuscular junction. Finally, we find that ActRIIB:ALK4-Fc enhances effects of dystrophin rescue therapy on dystrophin expression levels and skeletal muscle endurance in a murine model of DMD.

\section{Results}

ActRIIB:ALK4-Fc inhibits Smad3 signaling but does not inhibit the activity of BMP9. We engineered the novel heterodimeric fusion protein ActRIIB:ALK4-Fc to target multiple TGF- $\beta$ superfamily ligands implicated in muscle homeostasis. In Figure 1A, the domain structure of ActRIIB:ALK4-Fc is contrasted with that of ActRIIB-Fc homodimer. We used surface plasmon resonance (SPR) to compare the ligand binding profiles of these two fusion proteins. The resulting representative sensorgrams and summary of the data in Figure $1 \mathrm{~B}$ and Table 1 show that these proteins share similarly high affinities for GDF8, activin A, activin B, and GDF11 - all ligands that inhibit muscle growth. The equilibrium dissociation constants $\left(K_{D}\right)$ ranged from approximately $4.9 \mathrm{pM}$ to $70.7 \mathrm{pM}$ for ActRIIB-Fc and $5.4 \mathrm{pM}$ to $124 \mathrm{pM}$ for ActRIIB:ALK4-Fc, with dissociation rate constants $\left(k_{d}\right)$ ranging from approximately $6.1 \times 10^{-5} \mathrm{~s}^{-1}$ to $1.6 \times 10^{-4} \mathrm{~s}^{-1}$ for ActRIIB-Fc and from $6.6 \times 10^{-5} \mathrm{~s}^{-1}$ to $2.6 \times 10^{-4} \mathrm{~s}^{-1}$ for ActRIIB:ALK4-Fc. We next evaluated the ability of ActRIIB:ALK4-Fc to inhibit ligand-induced Smad2/3 signaling in C2C12 mouse myoblast cells. Compared with untreated cells, C2C12 cells treated separately with activin A, GDF8, or GDF11 showed increased levels of Smad3 phosphorylation, including increased levels of nuclear signal, whereas cotreatment with ActRIIB:ALK4-Fc prevented Smad3 phosphorylation in each case (Supplemental Figure 1, A-D; supplemental material available online with this article; https://doi. org/10.1172/JCI138634DS1). These results indicate that, like homodimeric ActRIIB-Fc (23), ActRIIB:ALK4-Fc binds activin A, activin B, GDF8, and GDF11 with high affinity under cell-free conditions and effectively inhibits Smad3 pathway activation by these ligands in a cellular context in vitro.
We next investigated potential interaction between ActRIIB:ALK4-Fc and BMP9, because inhibition of BMP9 might have been responsible for epistaxis and telangiectasia seen previously in a clinical study of ActRIIB-Fc (22). We hypothesized that BMP9, which signals through receptor complexes containing the type I receptor ALK1 (24), would fail to stably bind ActRIIB:ALK4-Fc and thus the vascular side effects seen with ActRIIB-Fc would be avoided. SPR sensorgram data in Figure 1B confirm that ActRIIB:ALK4-Fc interacts only transiently with BMP9, in contrast with the stable binding observed between BMP9 and ActRIIB-Fc homodimer. ActRIIB-Fc bound to BMP9 with a $K_{D}$ of approximately $116.1 \pm 10.8 \mathrm{pM}$ and a slow off-rate of $6.61 \times 10^{-4} \pm 18.9 \times 10^{-6}$ $\mathrm{s}^{-1}$, whereas these parameters could not be determined for the transient interaction between ActRIIB:ALK4-Fc and BMP9 (Table 1). We then tested activity of ActRIIB:ALK4-Fc in vivo in a retinal outgrowth assay, which is largely dependent on BMP9-induced ALK1 activation (25). Retinal flat mounts from mice treated with ActRIIB-Fc or ALK1-Fc, a known antiangiogenic agent (26), revealed inhibition of vessel outgrowth by these proteins compared with saline (7.9\% and 5.6\%, respectively; Figure 1, C and D). In contrast, treatment of mice with ActRIIB:ALK4-Fc did not inhibit retinal vessel outgrowth. Together, these in vitro and in vivo results indicate that ActRIIB:ALK4-Fc - unlike ActRIIB-Fc - neither binds BMP9 nor inhibits BMP9-dependent vascularization. Thus, we have generated a selective heterodimeric fusion protein, based on a native ActRIIB-ALK4 receptor pair, that binds with high affinity to negative regulators of muscle mass but not to BMP9.

ActRIIB:ALK4-Fc increases muscle mass and function in WT mice. ActRIIB-Fc effectively induces systemic muscle hypertrophy under diverse conditions (27). To determine whether ActRIIB:ALK4-Fc exhibits similar activity in vivo, we first evaluated effects of ActRIIB:ALK4-Fc on skeletal muscle in WT C57BL/6 mice. ActRIIB:ALK4-Fc administered s.c. twice weekly for 4 weeks induced dose-dependent systemic increases in total body weight (Supplemental Figure 2A). Analysis by whole-body nuclear magnetic resonance (NMR) revealed that increased total lean mass was accompanied by a reduction in total fat (Supplemental Figure 3, A and B). The highest dose of ActRIIB:ALK4-Fc (10 mg/kg) caused a 12-fold increase in total lean mass and reduced total fat mass by $4 \%$ (Supplemental Figure 3, A and B) compared with vehicle. Examination of individual skeletal muscles revealed that ActRIIB:ALK4-Fc treatment caused a significant dose-dependent increase in muscle weight compared with vehicle (Supplemental Figure 2, B-E). The highest dose of ActRIIB:ALK4-Fc produced weight increases of $53 \%$ in the tibialis anterior (TA), 33\% in the gastrocnemius, $16 \%$ in the extensor digitorum longus, and 37\% in the quadriceps (Supplemental Figure 2, B-E).

We then examined the TA muscle to determine effects of ActRIIB:ALK4-Fc on muscle fiber size, fiber type, and strength. ActRIIB:ALK4-Fc increased the mean physiological cross-sectional area (pCSA) of the TA by 49\% (Supplemental Figure 2C). Histologic analysis verified that ActRIIB:ALK4-Fc treatment increased individual fiber diameter compared with vehicle (Supplemental Figure 2, D and E) and indicated that effects of ActRIIB:ALK4-Fc on muscle mass were not due to hyperplasia (Supplemental Figure 2I). Subsequent pulse-labeling experiments with puromycin revealed that ActRIIB:ALK4-Fc promotes generalized protein synthesis in muscle (Supplemental Figure 4A), in this case an 
A
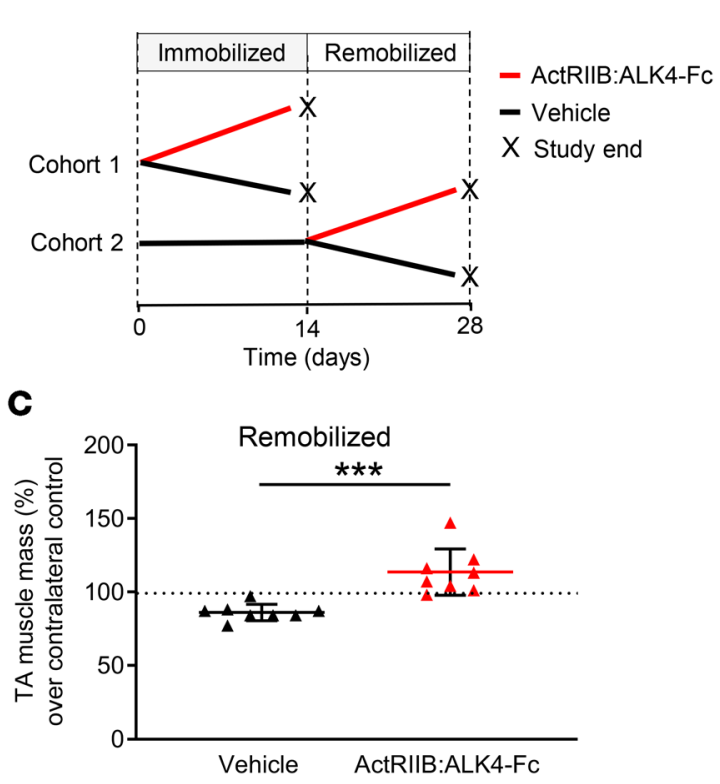
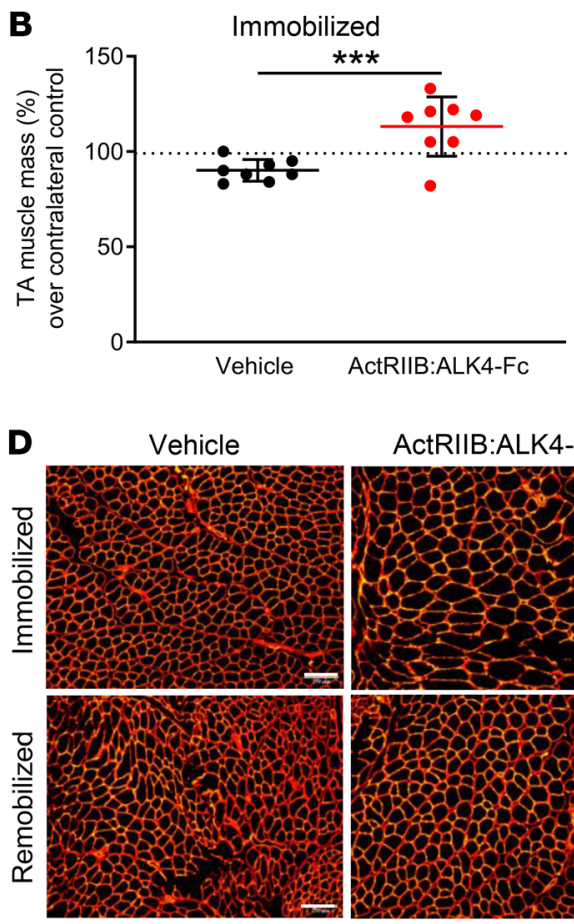

ActRIIB:ALK4-Fc

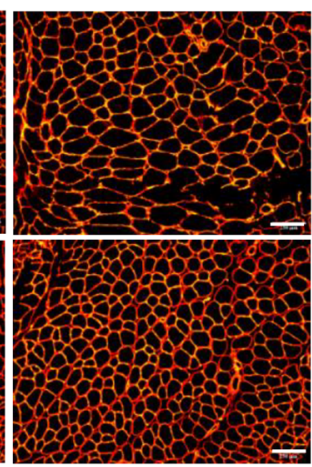

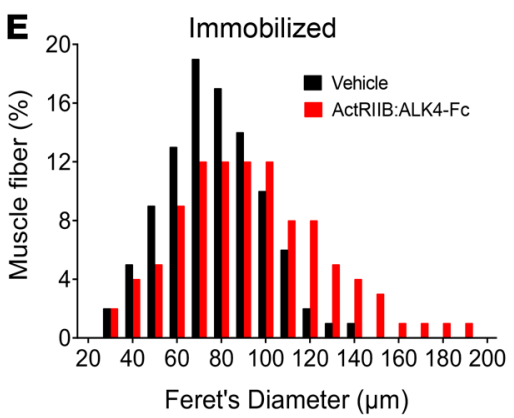

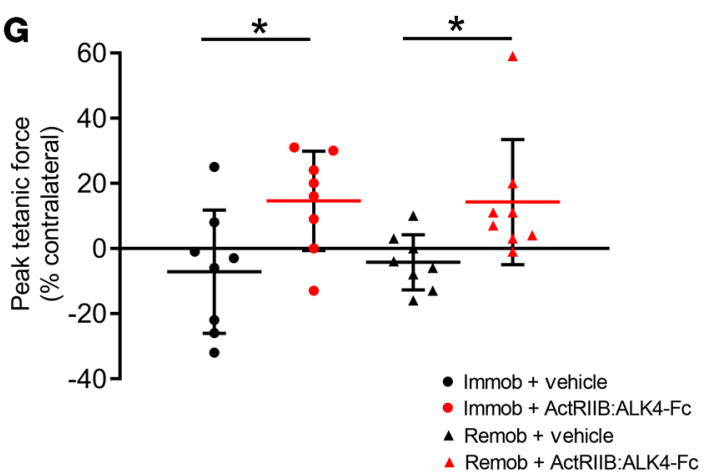

Figure 2. ActRIIB:ALIK4-Fc provides preventive and therapeutic benefits in a mouse model of disuse atrophy. (A) Experimental design depicting immobilized and remobilized cohorts of mice with hind-limb immobilization treated with ActRIIB:ALK4-Fc or vehicle (PBS) twice weekly for 14 days. (B) TA muscle mass after immobilization normalized to contralateral muscle. (C) TA muscle mass after remobilization normalized to contralateral muscle. (D) Laminin-stained sections of TA muscle. Scale bars: $250 \mu \mathrm{m}$. (E and F) Effect of treatment on the distribution of muscle fiber diameters in the immobilized and remobilized cohorts. (C) Peak tetanic force of the TA muscle after immobilization and remobilization normalized to contralateral muscle. Data are means \pm SEM ( $n=8$ per group). Group differences (B, C, and $\mathbf{C}$ ) were assessed by unpaired Student's $t$ test. ${ }^{*} P<0.05,{ }^{* *} P<0.001$.

increase of 1.7-fold $(P<0.05)$ compared with vehicle (Supplemental Figure 4B). ActRIIB:ALK4-Fc did not alter the fiber-type composition of the TA muscle based on immunodetection of myosin heavy chain (MHC) isoforms of IIa, IIb, or IIx fiber subtypes (Supplemental Figure 2F). Similarly, ActRIIB:ALK4-Fc did not alter fiber-type composition of the soleus, a muscle rich in the type I fiber subtype (Supplemental Figure 2J). Importantly, hypertrophic effects of ActRIIB:ALK4-Fc translated into increased TA muscle strength, as ActRIIB:ALK4-Fc augmented twitch force by $25 \%$ (Supplemental Figure 2G), peak tetanic force by $20 \%$ (Supplemental Figure 2H), and forelimb grip strength by $14 \%$ (Supplemental Figure 2K). Together, these data indicate that ActRIIB:ALK4-Fc treatment in WT mice produces muscle fiber hypertrophy accompanied by increased strength similar to effects observed previously with ActRIIB-Fc (27).
ActRIIB:ALK4-Fc exerts preventive and therapeutic effects in a mouse model of disuse atrophy. We next evaluated ActRIIB:ALK4-Fc as a potential preventive and therapeutic agent in a mouse model of disuse atrophy, a multidimensional myopathy typically resulting from sedentary behavior or periods of enforced immobilization. This experiment incorporated 2 cohorts of mice with hind-limb unloading (28), the first cohort receiving treatment with ActRIIB:ALK4-Fc or vehicle in a preventive manner beginning immediately after immobilization and the second cohort undergoing an immobilization phase followed by treatment with ActRIIB:ALK4-Fc or vehicle in a therapeutic manner beginning concurrently with remobilization (Figure 2A). Hind-limb immobilization for 2 weeks caused marked atrophy of TA muscle (10\% reduction in muscle mass), which was fully prevented by ActRIIB:ALK4-Fc (Figure 2B). Hind-limb remobilization for 2 weeks 

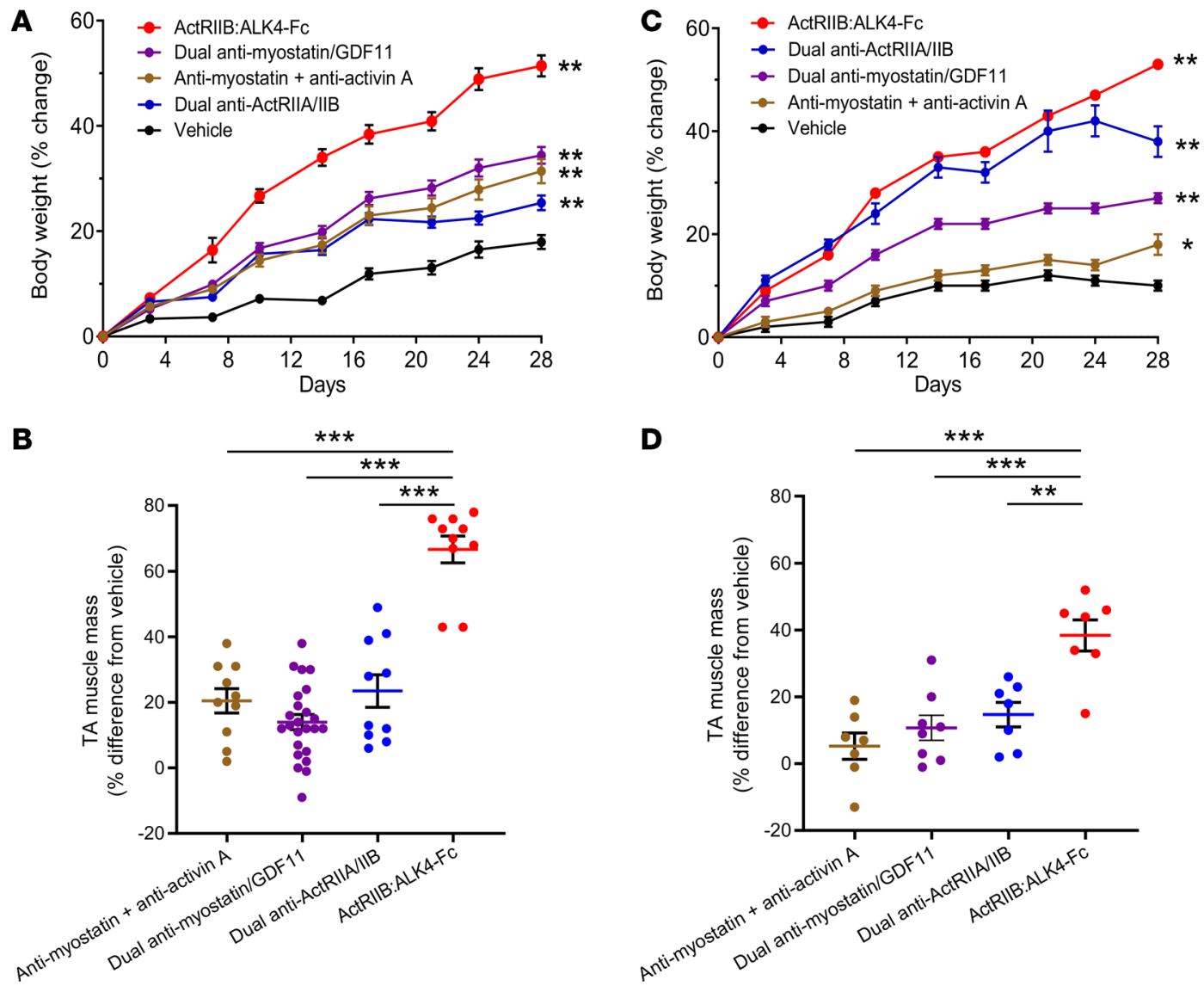

Figure 3. Multiligand inhibition with ActRIIB:ALK4-Fc robustly increases muscle mass in WT and DMD mice. Seven-week-old WT C57BL/6 mice or 5-week-old BL10.mdx mice were injected s.c. with ActRIIB:ALK4-Fc (10 mg/kg), a dual anti-ActRIIA/IIB antibody (10 mg/kg), a dual anti-myostatin/ GDF11 antibody (10 mg/kg), combined anti-myostatin plus anti-activin A antibodies (10 mg/kg $+10 \mathrm{mg} / \mathrm{kg}$ ), or vehicle (PBS) twice weekly for 28 days. (A) Percentage change in body weight over time in WT mice $(n=10-25)$. (B) Percentage difference in TA muscle mass compared with vehicle in WT mice ( $n=$ 10-29). (C) Percentage change in body weight over time in $m d x$ mice $(n=7-8)$. (D) Percentage difference in TA muscle mass compared with vehicle in $m d x$ mice $(n=5-18)$. Data are means \pm SEM. Group differences in $\mathbf{A}$ and $\mathbf{C}$ were assessed by 1-way ANOVA for repeated measures with Dunnett's adjustment. Group differences in $\mathbf{B}$ and $\mathbf{D}$ were assessed by 1-way ANOVA with Dunnett's adjustment. ${ }^{*} P<0.05,{ }^{* *} P<0.01,{ }^{* * *} P<0.001$.

was unable by itself to significantly reverse the prior atrophy of TA muscle; however, therapeutic treatment with ActRIIB:ALK4-Fc fully reversed the $14 \%$ loss in TA muscle mass caused by limb immobilization in this group (Figure 2C). Consistent with these changes in muscle mass, quantitative analysis of muscle fiber diameters in TA muscle revealed that ActRIIB:ALK4-Fc treatment produced a rightward shift in myofiber size histograms compared with vehicle in both the preventive (immobilized) and therapeutic (remobilized) cohorts (Figure 2, D-F). With vehicle treatment, peak tetanic force generated during isometric contractions of the TA was weaker than contralateral control in the immobilized and remobilized limbs by $7 \%$ and $4 \%$, respectively (Figure $2 \mathrm{G}$ ). However, muscle strength was more than fully restored by both preventive and therapeutic treatments with ActRIIB:ALK4-Fc (Figure $2 \mathrm{G}$ ). These data indicate that ActRIIB:ALK4-Fc improves muscle mass and strength under either preventive or therapeutic conditions in a rodent model of joint immobilization, which could have potential application in a clinical setting (29).

Multiligand inhibition with ActRIIB:ALK4-Fc increases muscle mass robustly in a mouse model of DMD. We then compared the efficacy of multiligand inhibition with ActRIIB:ALK4-Fc to that of other Smad2/3 pathway inhibitors, some of which have been investigated as potential treatments for muscular dystrophy (3033). For this experiment, we used WT mice and an $m d x$ mouse commonly used to model DMD (34). Seven-week-old BL10.mdx mice and age-matched WT mice were treated for 4 weeks with combined antibodies against GDF8 and activin A, an antibody with dual specificity for GDF8 and GDF11, an antibody with dual specificity for ActRIIA and ActRIIB, ActRIIB:ALK4-Fc, or vehicle. Compared with vehicle, all treatments increased total body weight progressively over the treatment period in WT and $m d x$ mice. However, ActRIIB:ALK4-Fc produced a greater increase in body weight than other treatments at comparable doses with the exception of the dual anti-ActRIIA/ActRIIB antibody, which exhibited an effect comparable to that of ActRIIB:ALK4-Fc in $m d x$ mice (Figure 3, A and C). In WT mice, ActRIIB:ALK4-Fc treatment produced a larger increase in TA muscle weight (67\%) than the dual anti-ActRIIA/ActRIIB antibody (24\%), the combination of antiGDF8 and anti-activin A antibodies (21\%), or the dual anti-GDF8/ GDF11 antibody (14\%; Figure 3B). In $m d x$ mice, ActRIIB:ALK4-Fc again produced the largest increase in TA weight (38\%), followed by the dual anti-ActRIIA/ActRIIB antibody (15\%), the dual anti- 

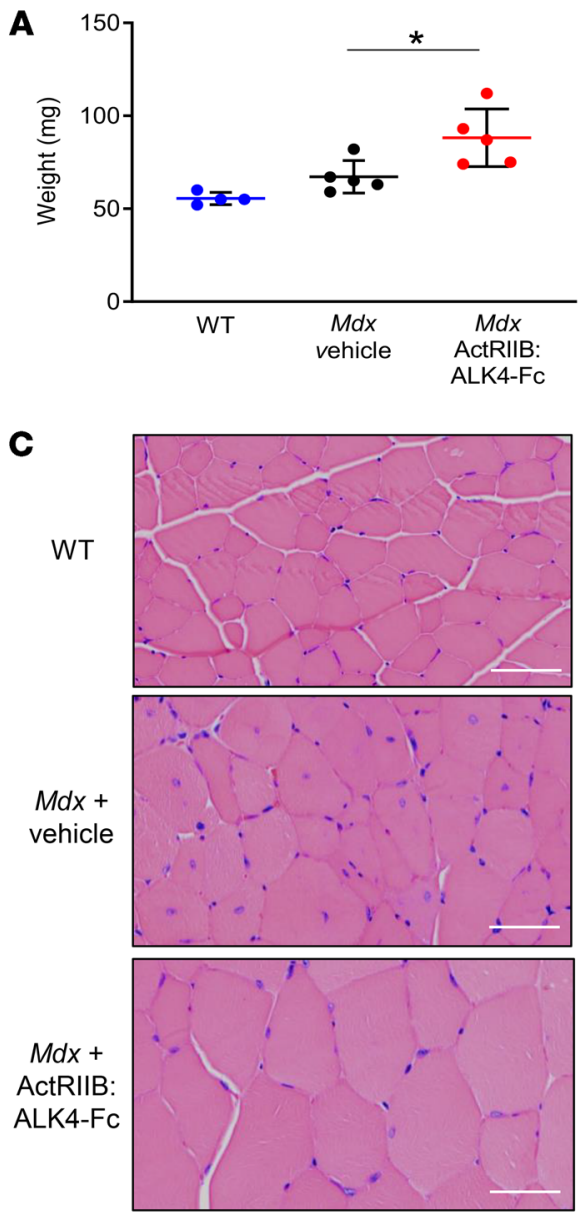

B
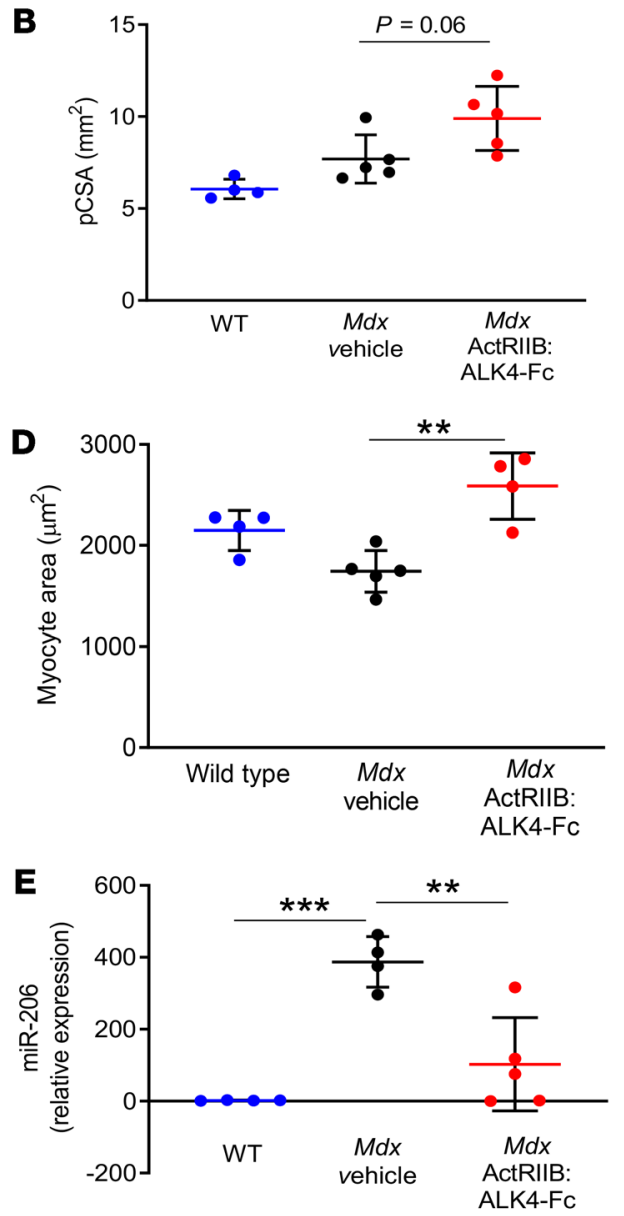

Figure 4. ActRIIB:ALK4-Fc induces muscle hypertrophy in an aged mouse model of DMD. Fourteen-month-old C57BL/6 WT and BL10.mdx mice were treated with ActRIIB:ALK4-Fc (10 mg/kg) or vehicle (PBS) twice weekly for 8 weeks. (A) TA weight. (B) TA physiological cross-sectional area ( $p C S A)$. (C) TA sections stained with H\&E. Scale bars: $50 \mu \mathrm{m}$. (D) TA myocyte area. (E) Serum levels of miR-206. Data are means \pm SEM ( $n=4-5$ per group). Group differences were assessed by 1-way ANOVA with Tukey's adjustment. ${ }^{*} P<0.05,{ }^{* *} P<0.01$, ${ }^{* * *} P<0.001$.
GDF8/GDF11 antibody (11\%), and the combination of anti-GDF8 and anti-activin A antibodies (5\%; Figure 3D), although these changes were consistently smaller than the corresponding effects in WT mice. These results indicate that, under normal and disease conditions, multiligand inhibition with ActRIIB:ALK4-Fc produces larger increases in skeletal muscle mass than these other inhibitors of Smad2/3 pathway signaling at comparable doses.

We next investigated whether ActRIIB:ALK4-Fc treatment exerts beneficial effects on muscle in aged $m d x$ mice, which display increasingly severe DMD-like signs over time (35). Fourteenmonth-old BL10.mdx mice were treated with ActRIIB:ALK4-Fc or vehicle for 8 weeks, and the TA was characterized as a representative skeletal muscle. Compared with vehicle, ActRIIB:ALK4-Fc treatment increased muscle mass by $31 \%$ and pCSA by $29 \%$ (Figure 4, A and B). Histologic examination of TA muscle from $m d x$ mice revealed that myofibers were heterogeneous in size owing to staggered cycles of damage and regrowth, although fibers from ActRIIB:ALK4-Fc-treated animals nevertheless appeared larger overall (Figure 4C). Quantification confirmed that ActRIIB:ALK4-Fc treatment increased mean cross-sectional area of TA myofibers by $49 \%$ compared with vehicle (Figure 4D) but did not affect the subcellular distribution of centralized nuclei (Supplemental Figure 5).

Additionally, we assessed the effect of ActRIIB:ALK4-Fc treatment on circulating levels of microRNAs previously implicated as biomarkers of muscle status in $m d x$ mice and DMD patients $(36,37)$, including miR-1, miR-21, miR-136b, miR-146a, and miR-206. As determined by quantitative PCR, serum levels of miR-206 were dramatically elevated (200-fold) in aged $m d x$ mice compared with WT mice, and ActRIIB:ALK4-Fc reduced the elevation of these levels by $74 \%$ (Figure $4 \mathrm{E}$ ). Levels of other microRNAs tested were found to be elevated in $m d x$ mice compared with WT and were similarly reduced by ActRIIB:ALK4-Fc treatment (Supplemental Figure 6). Among these was miR-146a, which has recently been shown to inhibit dystrophin translation (38). Together, our results indicate that multiligand inhibition with ActRIIB:ALK4-Fc increases muscle mass more effectively than comparable doses of agents targeting fewer Smad2/3 pathway ligands and reduces circulating levels of biomarkers implicated in impaired muscle function in the $m d x$ mouse model even at an advanced stage of disease progression.

ActRIIB:ALK4-Fc alleviates acute muscle fibrosis in a mouse model of DMD. Since DMD patients are afflicted by extensive fibrosis in skeletal muscle (39), we next investigated whether ActRIIB:ALK4-Fc can ameliorate fibrosis in an acute injury model (40). Endomysial fibrosis was established by daily micropunctures in TA muscles of BL10.mdx mice, and injured mice were then treated therapeutically for 4 weeks with either ActRIIB:ALK4-Fc or vehicle. As determined by staining with Picrosirius red, collagen fibers were more extensively distributed in muscle from injured $m d x$ mice than in uninjured mice, and ActRIIB:ALK4-Fc treatment restored collagen staining to levels characteristic of 
A

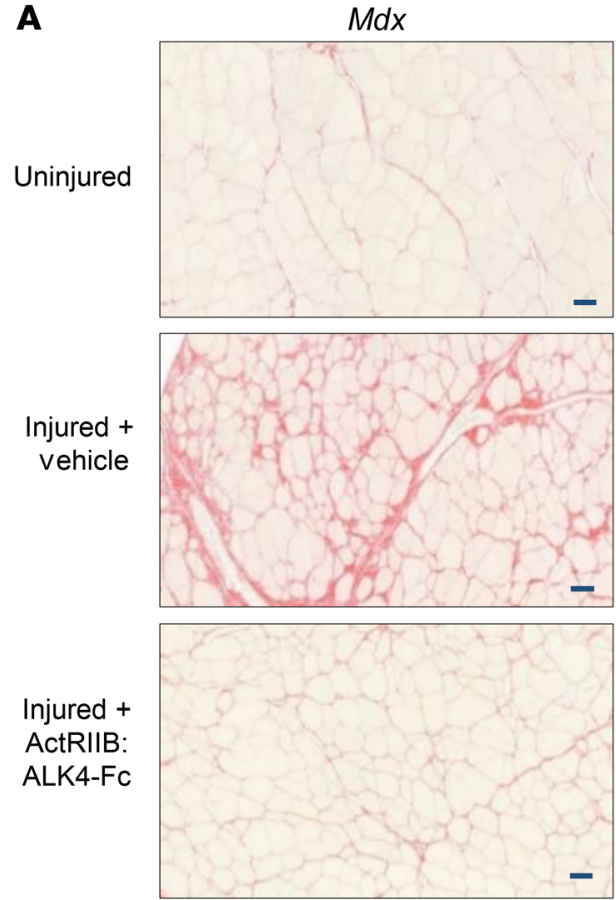

B
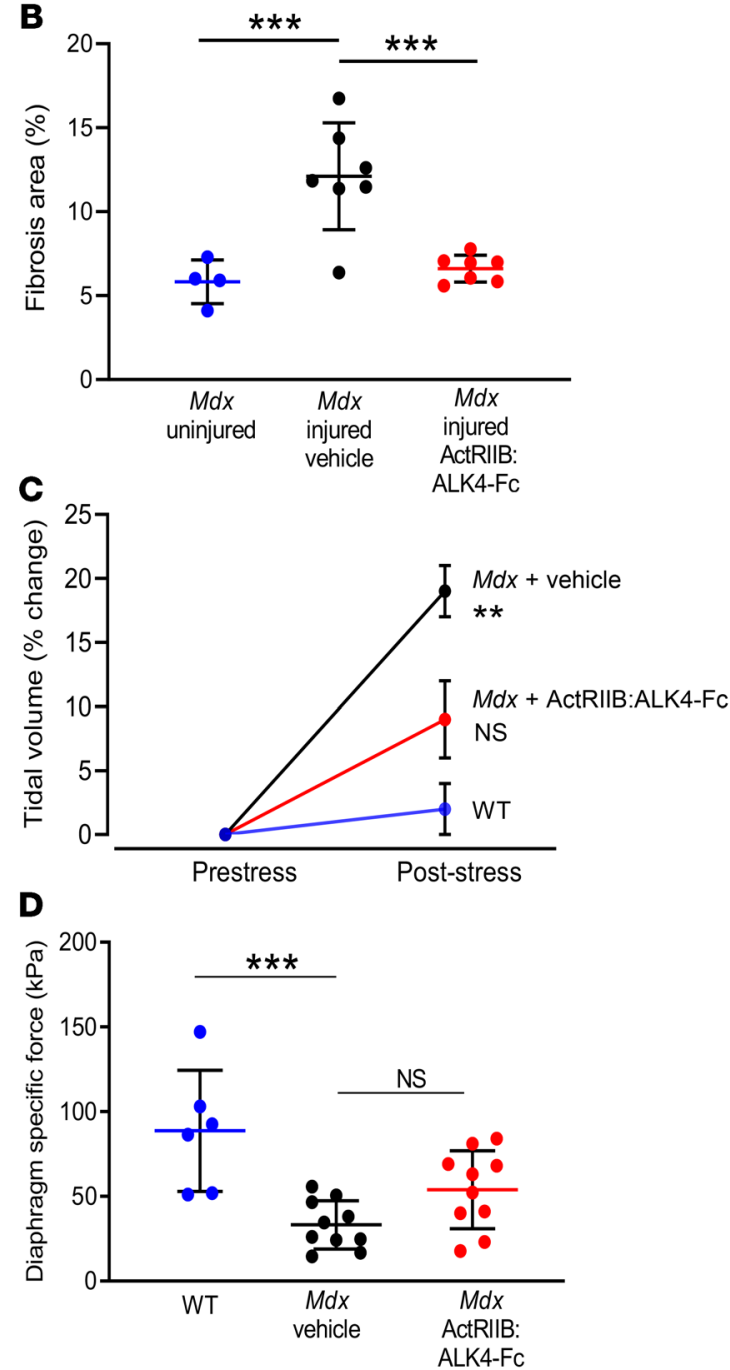

Figure 5. ActRIIB:ALK4-Fc alleviates acute fibrosis in a mouse model of DMD. Five-week-old BL10.mdx mice with mechanically induced fibrosis in the TA muscle were treated with ActRIIB:ALK4-Fc (10 mg/kg) or vehicle (PBS) twice weekly for 4 weeks. (A) TA muscle sections stained with Picrosirius red. Scale bars: $50 \mu \mathrm{m}$. (B) Percentage area in TA sections occupied by fibrotic tissue. Data are means \pm SEM $(n=4-7)$. Group differences were assessed by 1-way ANOVA with Tukey's adjustment. ${ }^{* *} P<0.001$. For respiratory studies, 7-month-old D2.mdx mice were injected s.c. with ActRIIB:ALK4-Fc (10 mg/kg) or vehicle (PBS) twice weekly for 4 weeks. (C) Percentage change in tidal volume after exposure to hypoxic stress $\left(10 \% O_{2}\right)$ for 30 minutes. Data are means \pm SEM $(n=3-5$ per group). Group differences were assessed by 1-way ANOVA with Tukey's adjustment. ${ }^{*} P<0.01$ vs. WT. (D) Specific force generated by diaphragm muscle ex vivo. Data are means \pm SEM ( $n=6-10$ per group). Group differences were assessed by 1-way ANOVA with Tukey's adjustment. ${ }^{* * *} P<0.001$.

uninjured controls (Figure 5, A and B). These data indicate that therapeutic treatment with ActRIIB:ALK4-Fc can reverse collagen deposition associated with fibrosis caused by repetitive muscle injury in $m d x$ mice.

We then investigated whether ActRIIB:ALK4-Fc can improve respiratory function, since DMD patients suffer from progressive hypoventilation due to myofiber injury and collagen deposition in respiratory skeletal muscles (41). We used the D2.mdx mouse model of DMD, as its diaphragm is more severely impaired than that of the BL10. $m d x$ strain and more closely resembles pathology in patients (42). In WT mice, tidal volume under hypoxic stress to mimic hypoventilation in patients was virtually unchanged $(2 \%)$ from the prestress baseline, whereas tidal volume in D2.mdx mice treated with vehicle increased by $19 \%$ under hypoxic stress (Figure 5C). ActRIIB:ALK4-Fc treatment in D2.mdx mice better pre- served tidal volume under hypoxic stress (9\% increase), but the difference from vehicle treatment did not reach significance $(P$ $=0.07$ ) (Figure 5C). Measurement of diaphragm contractility ex vivo confirmed that specific force generated by the diaphragm of vehicle-treated D2.mdx mice was significantly reduced compared with that of WT mice. Treatment of D2.mdx mice with ActRIIB:ALK4-Fc improved diaphragm contractility by more than $60 \%$ compared with vehicle, but this increase was not statistically significant (Figure 5D).

ActRIIB:ALK4-Fc improves integrity and function of the neuromuscular junction in mouse models of diverse neuromuscular diseases. The neuromuscular junction (NMJ) plays an obligatory role in muscle contraction. Since neuromuscular transmission is impaired in DMD patients and mouse models of the disease partly because of NMJ abnormalities arising from dystrophin deficiency 
A

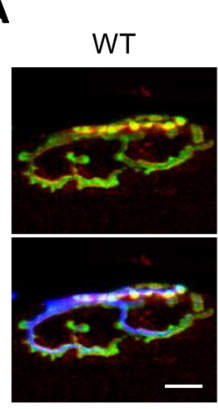

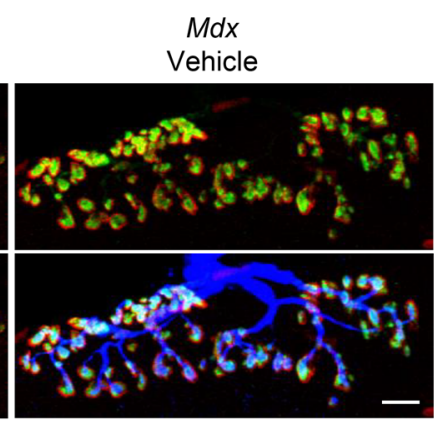

Mdx
$M d x$

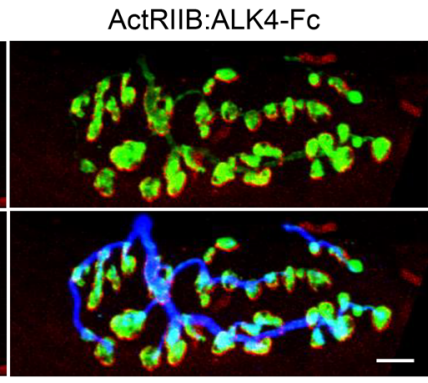

B
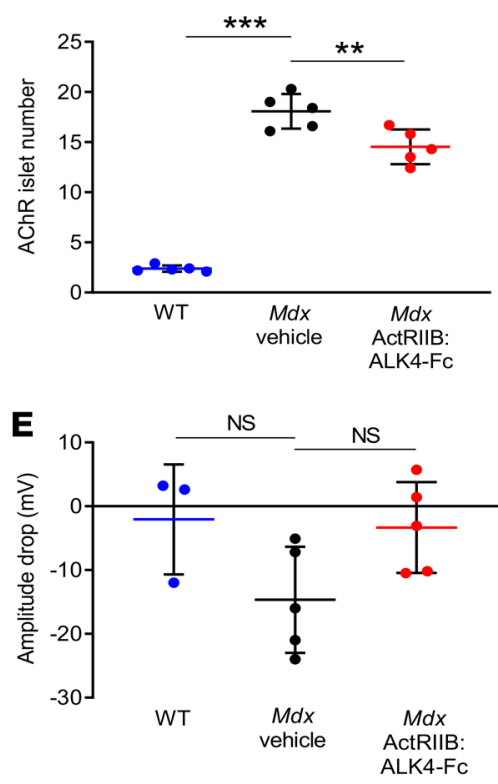

H Pretreatment Post-treatment

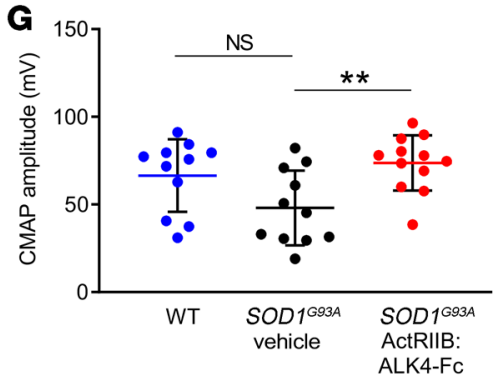

D
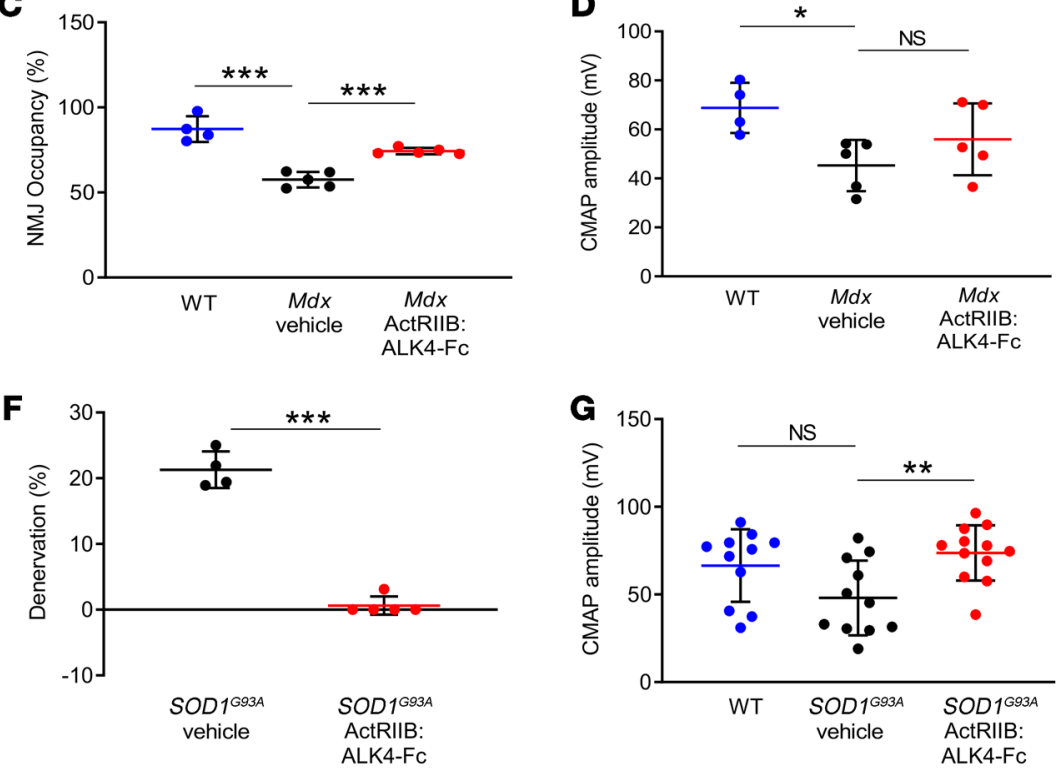

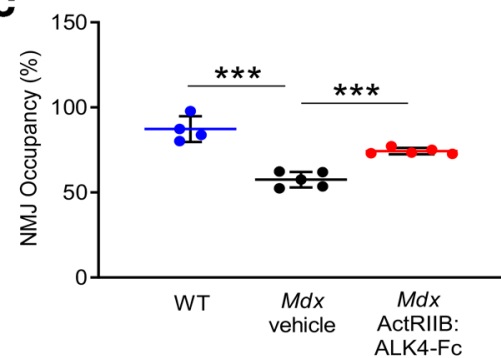

ALK4-FC

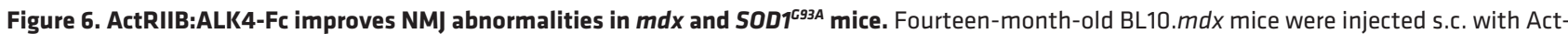
RIIB:ALK4-Fc (10 mg/kg) or vehicle (PBS) twice weekly for 8 weeks. (A) En face views of NMJs from WT mice and mdx mice treated with ActRIIB:ALK4-Fc or vehicle. Immunofluorescent confocal microscopic images indicate AChR (labeled by $\alpha$-bungarotoxin, red), vesicular acetylcholine transporter (VAChT, green), and their overlap (yellow). Blue in bottom panels indicates nerve fibers. Scale bars: $20 \mu \mathrm{m}$. (B) AChR islet number $(n=5)$. (C) Percentage NMJ occupancy $(n=4-5)$. (D) CMAP amplitude $(n=4-5)$. (E) Amplitude drop $(n=3-5)$. Data (B-E) are means \pm SEM. Group differences were assessed by $1-$ way ANOVA with Tukey's adjustment. ${ }^{*} P<0.05$. ${ }^{* *} P<0.01,{ }^{* *} P<0.001$. Five-week-old C57BL/6 WT mice or SOD1 ${ }^{\text {C93A }}$ mice were injected s.c. with ActRIIB:ALK4-Fc $(10 \mathrm{mg} / \mathrm{kg}$ ) or vehicle (PBS) twice weekly for 8 weeks, and TA muscles were analyzed. (F) Percentage of NMJs denervated $(n=4-5)$. (G) CMAP amplitude $(n=11-12)$. (H) Percentage change in ankle dorsiflexion $(n=6)$. Data are means \pm SEM. Group differences were assessed in $\mathbf{G}$ by 1 -way ANOVA with Tukey's adjustment and in $\mathbf{F}$ and $\mathbf{H}$ by unpaired Student's $t$ test. ${ }^{* *} P<0.01,{ }^{* *} P<0.001$.

(43-45), we next investigated whether ActRIIB:ALK4-Fc treatment improves NMJ morphology or function in aged $m d x$ mice. At NMJs from TA muscle of WT mice under normal conditions, clusters of acetylcholine receptors (AChRs) were tightly arranged with other NMJ components in characteristic "pretzel-like" arrays on the postsynaptic membrane as previously described (ref. 46 and Figure 6A). In striking contrast, NMJs from $m d x$ mice treated with vehicle displayed fragmentation of AChR clusters into discontinuous structures (islets) as previously reported $(45,47)$. Interestingly, NMJs in $m d x$ mice treated with ActRIIB:ALK4-Fc exhibited an intermediate morphology (Figure 6A), which prompted us to examine ActRIIB:ALK4-Fc effects quantitatively. The number of AChR islets in NMJs was increased 7.6-fold in vehicle-treated $m d x$ mice compared with WT mice, and ActRIIB:ALK4-Fc treatment significantly blunted this increase (Figure 6B). NMJ occupancy - defined as the ratio of footprint occupied by presynaptic vesicles to that of the underlying postsynaptic membrane (48) - was reduced by one-third in vehicle-treated $m d x$ mice compared with WT mice, and ActRIIB:ALK4-Fc treatment significantly improved this parameter (Figure 6C). These results indicate that treatment with ActRIIB:ALK4-Fc exerts a partially normalizing influence on NMJ morphology in $m d x$ mice.

We then used electrophysiologic analysis to determine whether these morphologic changes induced by ActRIIB:ALK4-Fc in $m d x$ mice were accompanied by functional improvements. In vehicle-treated $m d x$ mice compared with WT mice, there was a significant reduction by one-third in the amplitude of compound muscle action potentials (CMAPs) and a 7-fold reduction in amplitude drop during repetitive stimulation (Figure 6, D and E). ActRIIB:ALK4-Fc treatment in $m d x$ mice produced a trend toward improvement for CMAP amplitude and particularly amplitude drop during repetitive nerve stimulation (Figure 6, D and E). Although not statistically significant, these electrophysiologic effects raise the intriguing possibility that ActRIIB:ALK4-Fc 

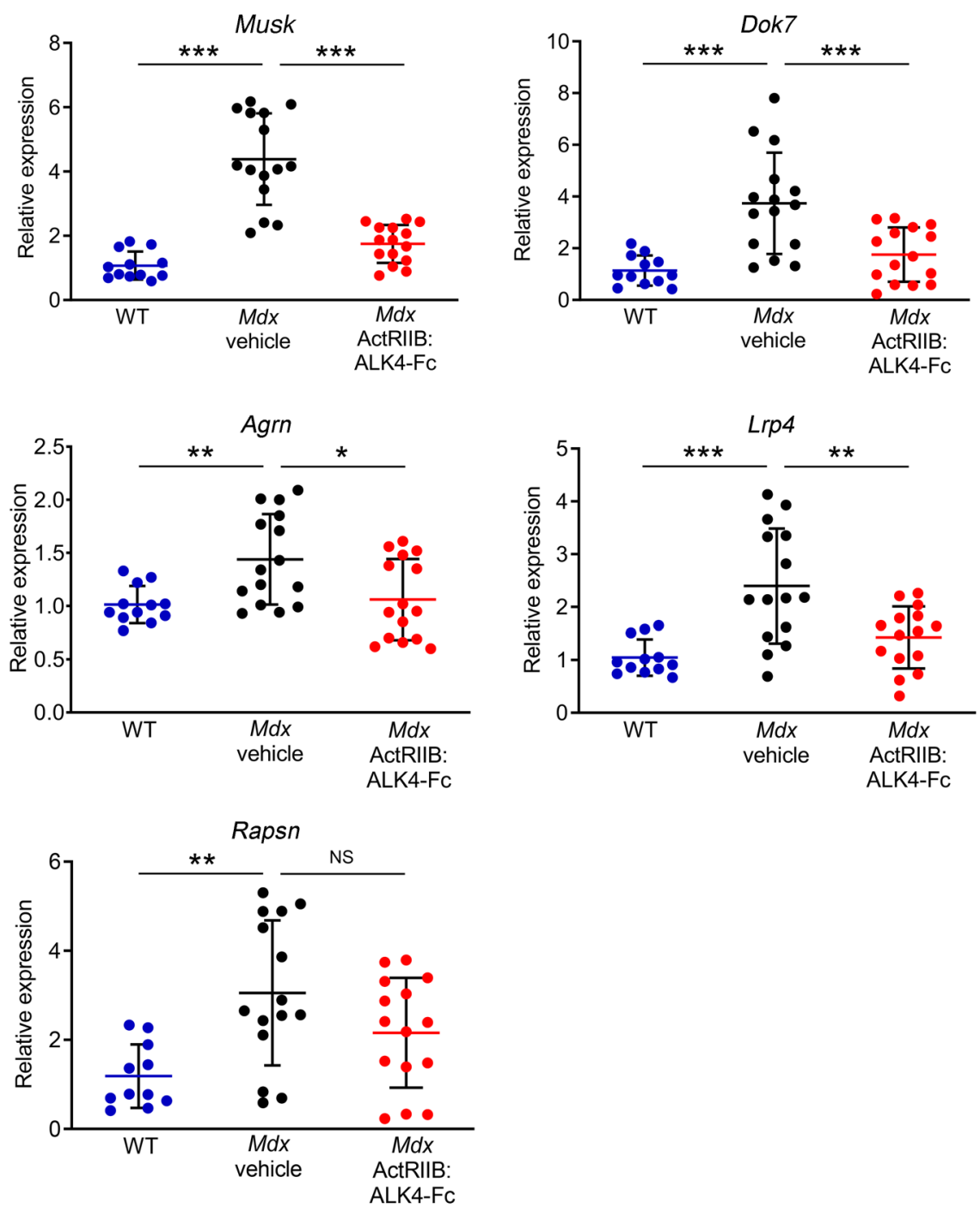

Figure 7. ActRIIB:ALIK4-Fc reverses elevated expression of NMJ genes in aged $m d x$ mice. Fourteenmonth-old C57BL/6 WT and mdx mice were injected s.c. with ActRIIB:ALK4-Fc (10 mg/kg) or vehicle (PBS) twice weekly for 4 weeks. Expression of key NMJ genes was determined in TA muscle by quantitative PCR and normalized to Actb. Data are means \pm SEM ( $n=11-15$ per group). Group differences were assessed by 1 -way ANOVA with Tukey's adjustment. ${ }^{*} P<0.05$, ${ }^{* *} P<0.01$, ${ }^{* *} P<0.001$. comparison with WT animals, and ActRIIB:ALK4-Fc treatment in SOD1 ${ }^{693 A}$ mice completely prevented this loss (Figure 6G). We evaluated the effect of a longer period of ActRIIB:ALK4-Fc treatment (7 weeks) on muscle function in $S O D 1^{G 93 A}$ mice and found that this treatment almost completely protected against the marked decline in ankle dorsiflexion torque observed in vehicle-treated controls (Figure 6H). These data indicate that ActRIIB:ALK4-Fc treatment protects against muscle denervation, improves nerve transmission, and increases muscle strength in this presymptomatic model of neurodegenerative disease. Together with results from our $m d x$ studies, these findings provide evidence that ActRIIB:ALK4-Fc treatment improves NMJ structure and function in models of distinct myopathic and neuropathic diseases.

Elevated expression of key NMJ genes is reversed by ActRIIB:ALK4-Fc in an aged mouse model of DMD. To better understand how ActRIIB:ALK4-Fc affects NMJ morphology and function, we investigated effects of this agent on expression of genes considered critical for formation and maintenance of neuromuscular synapses (49). Expression levels of Agrn (encoding agrin), Musk (muscle-specific kinase), Lrp4 (LDL receptor-related protein-4), Dok7 (downstream of tyrosine kinase 7), and Rapsn (receptor-associated protein of the synapse) were all significantly elevated in excised TA muscle (and associated neural tissue) of $m d x$ mice compared with WT mice (Figure 7), which likely represents a homeostatic response to diminished muscle fiber activation. Importantly, ActRIIB:ALK4-Fc treatment reversed the elevated expression of these mRNAs significantly in $m d x$ mice, except for Rapsn (Figure 7). The concordant effects increases postsynaptic sensitivity to acetylcholine in $m d x$ mice. Together, our results indicate that ActRIIB:ALK4-Fc alleviates morphologic abnormalities of the NMJ in this mouse model of DMD and raise the possibility of accompanying functional benefits that need to be investigated further.

We next investigated whether ActRIIB:ALK4-Fc alters neuromuscular transmission in a mouse model of amyotrophic lateral sclerosis (ALS), a disease with a primarily neurodegenerative etiology. We treated 5-week-old $S O D 1^{693 A}$ mice with ActRIIB:ALK4-Fc or vehicle for 3 weeks and examined parameters of neuromuscular transmission in the TA muscle. In vehicle-treated $S O D 1^{693 A}$ mice at this presymptomatic stage, approximately one-fifth of NMJs were denervated, whereas treatment with ActRIIB:ALK4-Fc essentially prevented NMJ denervation (Figure 6F). At innervated NMJs from vehicle-treated $S O D$ $1^{693 A}$ mice, there was a $29 \%$ reduction in CMAP amplitude in of ActRIIB:ALK4-Fc on this set of genes likely reflect a partial normalization of NMJ function and implicate Smad2/3 pathway ligands in the regulation of $\mathrm{NMJ}$ homeostasis, either directly or indirectly, under these disease conditions.

Combined treatment with ActRIIB:ALK4-Fc and M12-PMO synergistically improves muscle function in an aged mouse model of DMD. Therapeutics that act through antisense-mediated exon skipping, such as the recently approved Exondys51, are novel approaches to overcome dystrophin deficiency in DMD (50). However, exon skipping is considered insufficient to fully restore muscle strength or protect against contraction-induced injury $(51,52)$. We hypothesized that using the phosphorodiamidate morpholino oligomer M12-PMO (53) to restore dystrophin expression in combination with ActRIIB:ALK4-Fc to increase muscle mass would improve muscle function in $m d x$ mice, perhaps synergistically as a result of their seemingly complementary mechanisms of action. Thirteen- 
A

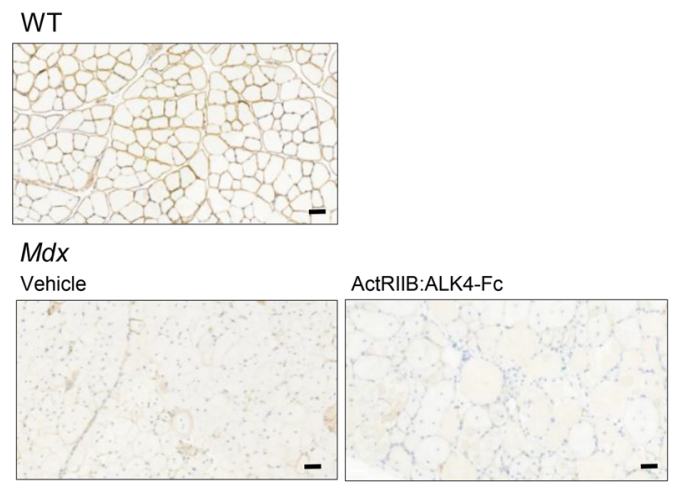

M12-PMO ActRIIB:ALK4-FC + M12-PMO
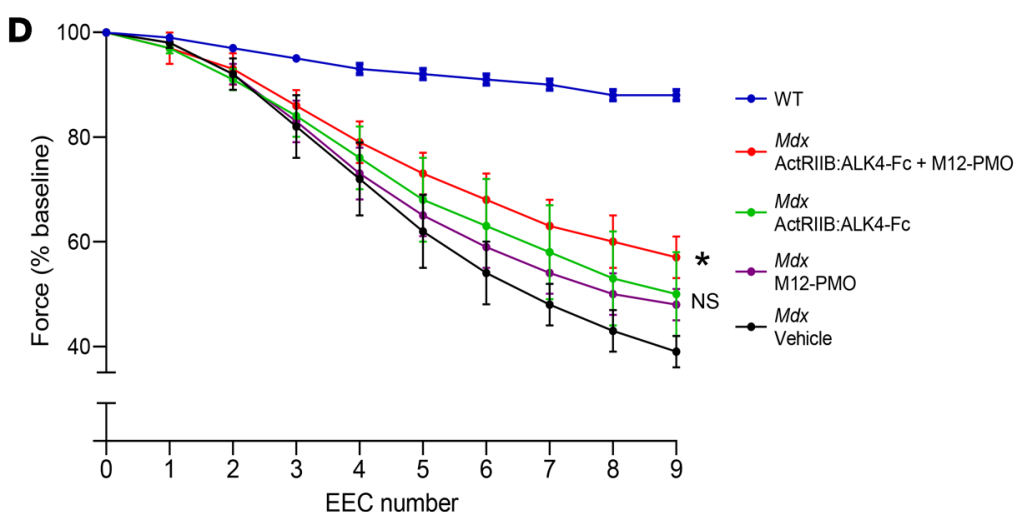

B

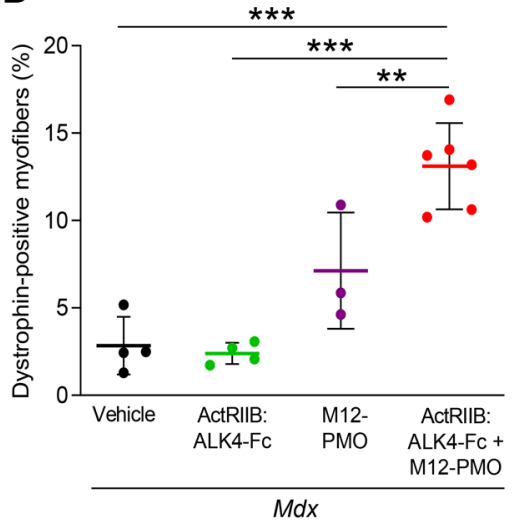

C
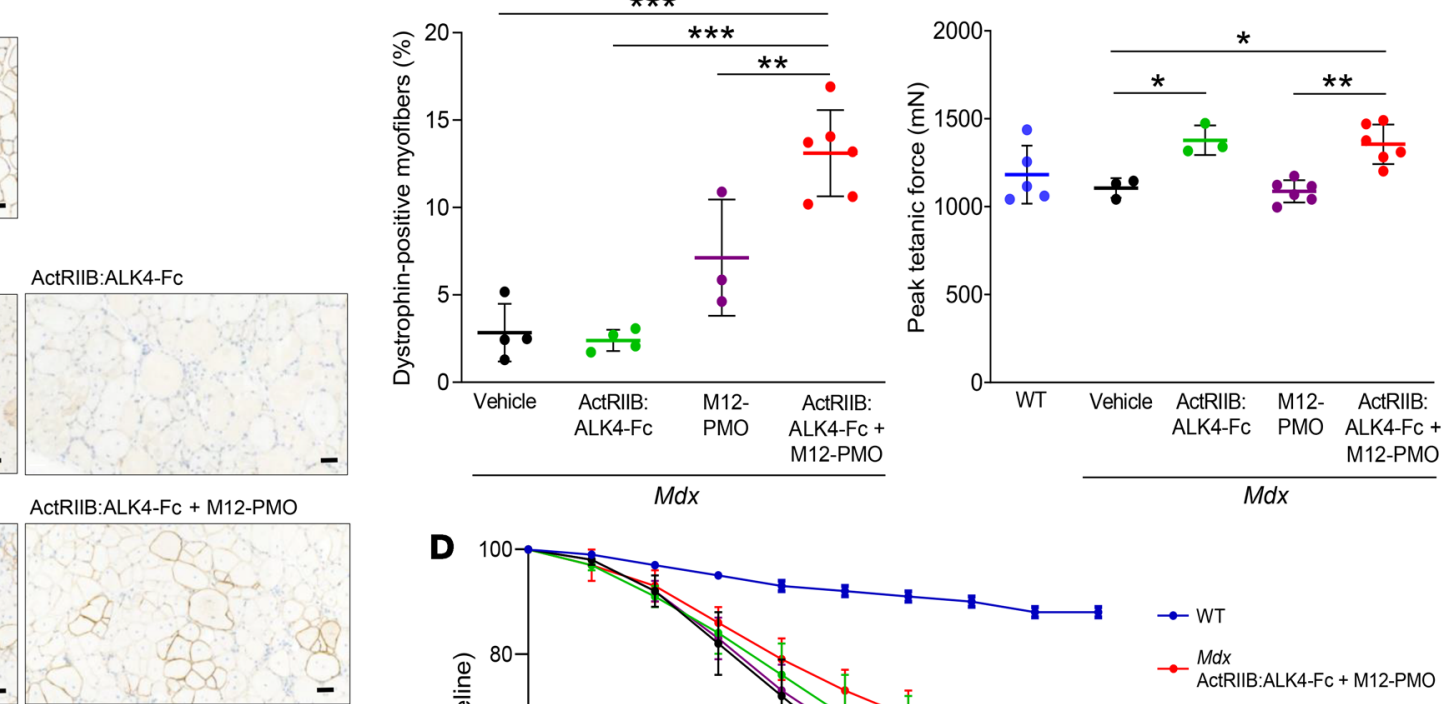

Figure 8. Combined treatment with ActRIIB:ALK4-Fc and M12-PMO improves muscle function in aged $m d x$ mice. Thirteen-month-old BL10.mdx mice were injected with ActRIIB:ALK4-Fc (10 mg/kg) or vehicle (PBS) s.c. twice weekly, M12-PMO (25 mg/kg) i.p. once weekly, or a combination of ActRIIB:ALK4-Fc twice weekly and M12-PMO once weekly for 4 weeks. (A) Dystrophin immunostaining in TA muscle sections. Scale bars: $50 \mu \mathrm{m}$. (B) Percentage of TA muscle fibers that were dystrophin positive. (C) Peak tetanic force generated by TA muscle. (D) Force generated during repeated eccentric exercise contractions (EEC) by TA muscle. Data are means \pm SEM ( $n=3-6$ per group). Group differences in $\mathbf{B}$ and $\mathbf{C}$ were assessed by 1-way ANOVA with Tukey's adjustment. ${ }^{*} P<0.05,{ }^{* *} P<0.01,{ }^{* *} P<0.001$. Group differences in $\mathbf{D}$ were assessed by repeated-measures 1 -way ANOVA with Tukey's adjustment. ${ }^{*} P<0.05$ vs. $m d x+$ vehicle.

month-old BL10.mdx mice were treated with ActRIIB:ALK4-Fc or M12-PMO, alone or in combination, over a 4-week period. Immunohistochemical staining confirmed that dystrophin was abundant in TA muscle fiber membranes of WT mice, clearly deficient in $m d x$ mice (regardless of ActRIIB:ALK4-Fc treatment), and partially restored by M12-PMO treatment (Figure 8A). Based on quantitative analysis, treatment with ActRIIB:ALK4-Fc did not alter the occurrence of dystrophin-positive myofibers, treatment with M12-PMO increased such myofibers by $7 \%$, and combined treatment with ActRIIB:ALK4-Fc and M12-PMO increased such myofibers by $13 \%$, all compared with vehicle (Figure 8B). These findings were further supported by Western blot analysis using an anti-dystrophin antibody (Supplemental Figure 7), which demonstrated synergistic effects of combined treatment on dystrophin protein abundance within TA muscle. This synergistic increase in dystrophin-positive fibers after combination treatment may be due to an ability of ActRIIB:ALK4-Fc to increase overall protein synthesis, as observed in WT mice (Supplemental Figure 4), and an ability of this agent to reduce expression of dystrophin translational inhibitors, such as its effect on miR-146a levels in $m d x$ mice (Supplemental Figure 5A). In an evaluation of functional endpoints, both ActRIIB:ALK4-Fc monotherapy and combina- tion therapy with ActRIIB:ALK4-Fc and M12-PMO increased peak tetanic force generated by the TA muscle in $m d x$ mice compared with vehicle or WT mice, whereas M12-PMO monotherapy was ineffective (Figure 8C). Importantly, combination therapy with ActRIIB:ALK4-Fc and M12-PMO provided a significant benefit in force generation during repeated eccentric contractions of the TA muscle in $m d x$ mice compared with vehicle, unlike either monotherapy (Figure 8D). Together, these results indicate that ActRIIB:ALK4-Fc could potentially be beneficial as an adjunct to dystrophin restoration therapy in patients with DMD because of complementary effects exerted by the 2 classes of agents.

\section{Discussion}

Here, we show that ActRIIB:ALK4-Fc, an inhibitor of Smad2/3 pathway signaling with novel ligand selectivity, improves skeletal muscle mass, muscle function, and disease comorbidities in murine models of NMDs. ActRIIB:ALK4-Fc displays activity as monotherapy across a range of modeled disorders, including disuse atrophy resulting from limb immobilization, the neurodegenerative disease ALS, and the inherited myopathy DMD - and in the latter case also enhances efficacy of a dystrophin rescue agent as part of combination therapy. Although limb muscle weakness 
and atrophy are the most prominent characteristics of neuromuscular diseases, secondary complications such as muscle fibrosis and respiratory insufficiency can impact survival and quality of life (54). We hypothesized that some of these functional deficits might be alleviated by qualitative improvements in muscle and find that ActRIIB:ALK4-Fc alleviates respiratory dysfunction and acute fibrosis in skeletal muscle in mouse models of DMD. Additionally, ActRIIB:ALK4-Fc improves the structural and functional integrity of the NMJ in mouse models of DMD and ALS, which to our knowledge is the first time this has been demonstrated using an inhibitor of Smad2/3 pathway signaling.

An important finding in our study is that combined inhibition of multiple Smad2/3 pathway ligands with ActRIIB:ALK4-Fc outperforms comparable doses of selective inhibitors of these ligands as a strategy for inducing skeletal muscle growth under pathologic conditions. Our results are consistent with a growing recognition that GDF8 and activins together exert an inhibitory homeostatic influence on muscle mass, perhaps synergistically and with differences between species $(18,19,32,55)$. Based mainly on studies in WT mice, others have proposed that concurrent inhibition of activin A and GDF8 with cognate antibodies increases muscle mass and force generation just as effectively as multiligand inhibition with ActRIIB-Fc (19). However, we found here in an $m d x$ mouse model of DMD, and in WT mice, that multiligand inhibition with ActRIIB:ALK4-Fc produces significantly larger increases in muscle mass than antibody-based ligand inhibition and even dual immunoneutralization of ActRIIA and ActRIIB. The ability of ActRIIB:ALK4-Fc to outperform an antibody with dual specificity for ActRIIA and ActRIIB is noteworthy and consistent with the possibility that one or more Smad1/5/8 pathway ligands normally signaling through these type II receptors - but not sequestered by ActRIIB:ALK4-Fc - promote muscle growth (56). A theoretical advantage of multiligand inhibition is that it may be effective despite a potential shift in the relative contributions of individual Smad2/3 pathway activators under disease conditions (17), although the Smad2/3 pathway inhibitory agents that we tested display nearly the same rank order of efficacy in young $m d x$ mice as under normal conditions, perhaps owing to the early stage of disease progression. Since contractile muscle mass is positively correlated with muscle function (57), robust increases in muscle mass such as those achieved with ActRIIB:ALK4-Fc treatment may be required to surpass the threshold for clinically meaningful efficacy in NMDs.

ActRIIB:ALK4-Fc treatment in mouse models produced beneficial changes in important comorbidities of neuromuscular disease. First, therapeutic treatment with ActRIIB:ALK4-Fc effectively reversed fibrotic changes induced acutely in skeletal muscle of an $m d x$ mouse model. Growing evidence indicates that the diaphragm in aged $m d x$ mice is significantly impacted by fibrosis (58), and systemic immunoneutralization of GDF8 reduces fibrosis in the diaphragm of aged $m d x$ mice (59), implicating this Smad2/3 pathway ligand in late-stage fibrosis. Our result is consistent with the previously reported ability of ActRIIB-Fc treatment to reverse preexisting fibrosis in skeletal muscle of aged $m d x$ mice (59) and the ability of follistatin treatment to reduce fibrosis caused by skeletal muscle injury in WT mice (60). Heterodimeric ActRIIB:ALK4-Fc, homodimeric ActRIIB-Fc, and endogenous follistatin monomers share the ability to sequester GDF8, activins, and GDF11. Although the foregoing antifibrotic effects of ActRIIB-Fc and follistatin were attributed mainly to their inhibition of $\operatorname{GDF} 8(60,61)$, activin inhibition by these agents and ActRIIB:ALK4-Fc might also contribute to similarities in their muscle activity profiles, since activins are implicated in muscle remodeling as well as inflammation generally $(62,63)$. Our present finding that ActRIIB:ALK4-Fc alleviates fibrosis in the acute micropuncture model warrants further investigation in models of advanced chronic muscular dystrophy. The micropuncture procedure has been reported to be a better model of prolonged fibrosis of skeletal muscle in younger $m d x$ mice compared with procedures based on chemical or surgical induction $(40,64)$. However, DMD patients display chronic fibrosis in skeletal muscle, which is better represented in aged $m d x$ mice $(42,59)$. Given that ActRIIB:ALK4-Fc treatment increased skeletal muscle mass and function in 12-month-old $m d x$ mice, it will be important to evaluate the effect of ActRIIB:ALK4-Fc treatment in aged mice with established fibrosis (59). Regarding respiratory function, ActRIIB:ALK4-Fc treatment was associated with trends toward better maintenance of tidal volume under hypoxic stress and increased specific force generated by the diaphragm in those mice. We speculate that the diaphragm of $m d x$ mice at this age is resistant to treatment because of loss of myofibers (58). Therefore, the trend toward improved respiratory function with ActRIIB:ALK4-Fc treatment may partially reflect improved function of intercostal or inspiratory accessory muscles (65).

NMJ abnormalities contribute significantly to the pathology of NMDs (66). The mature NMJ is a dynamic structure subject to remodeling based on bidirectional communication between muscle fibers and motor neurons that normally maintains proper alignment between presynaptic terminal boutons and components of the motor endplate $(43,46,67)$. Consistent with earlier studies $(45,48)$, we observed that AChR clusters in NMJs of aged mdx mice were discontinuous and fragmented, and poorly aligned with presynaptic NMJ components. Aged $m d x$ mice also displayed a functional deficit (reduction in CMAP amplitude) compared with age-matched WT mice. To our knowledge, the effects of ActRIIB:ALK4-Fc treatment on NMJ structure and associated muscle function in models of DMD and ALS are the first report of a muscle-targeted therapy causing beneficial remodeling of NMJ morphology and related improvement in NMJ function.

AChR clustering in the postsynaptic membrane of the NMJ is regulated by the agrin/MuSK/Lrp4 pathway (68-70). We found that genes encoding components of the MuSK signaling complex at the NMJ are upregulated in aged $m d x$ mice and are partially reversed by ActRIIB:ALK4-Fc treatment. Given evidence of reduced sensitivity to acetylcholine at neuromuscular synapses in DMD mice (45), we interpret coordinated upregulation of these genes to be a compensatory response of the muscle fiber to reduced stimulation under disease conditions. A similar association between reduced NMJ integrity and elevated expression of Musk and Lrp4 has been observed in sarcopenic rats (68, 71). A study in young $m d x$ mice found that expression of Agrn, Musk, Dok7, Rapsn, and Lrp4 was largely unchanged in comparison with WT (70), which suggests that their expression varies in a direct manner with disease severity - and thus NMJ integrity. Therefore, we speculate that partially normalized expression of these genes by ActRIIB:ALK4-Fc treatment reflects a reduced 
compensatory response secondary to improved myofiber physiology due perhaps to a shift in the balance between Smad2/3 signaling and Smad1/5/8 signaling.

ActRIIB:ALK4-Fc might improve NMJ structure and function in part by modulating the balance of muscle-derived retrograde signaling mediated by TGF- $\beta$ superfamily ligands. Studies in Drosophila indicate that retrograde trans-synaptic signaling by BMPs released from target muscle fibers or neurons supports NMJ growth, neurotransmission, and synaptic plasticity (72-74). Additionally, myoglianin (MYO), the Drosophila homolog of GDF8 and GDF11, was found to negatively regulate synaptic function and neuronal morphology, which affected both NMJ strength and composition (75). In the same study, GDF8 and GDF11 prevented synapse formation in cultured rat cortical neurons. Therefore, pharmacologic regulation of TGF- $\beta$ superfamily signaling could potentially promote NMJ integrity and function through indirect retrograde signaling in addition to direct effects on muscle fibers. In the present study, ActRIIB:ALK4-Fc treatment in a presymptomatic SOD1 ${ }^{G 93 A}$ mouse model of ALS preserved muscle innervation and improved neuromuscular transmission. In this case, ActRIIB:ALK4-Fc may increase production of muscle-derived neurotrophic factors or potentially regulate motor neuron function directly since BMPs can promote neuronal growth through direct actions $(76,77)$.

Dystrophin rescue therapies such as antisense-mediated exon skipping are promising treatments for muscular dystrophy but have yet to demonstrate functional benefit in clinical studies (13). Hence, there is increasing interest in combination therapies to optimize treatment efficacy for DMD, a strategy in which an agent intended to correct an underlying genetic deficiency would be combined with a second agent intended to synergistically enhance therapeutic efficacy by targeting muscle degeneration, fibrosis, or inflammation (78). In the present study, ActRIIB:ALK4-Fc monotherapy did not alter the prevalence of dystrophin-positive muscle fibers, yet in combination therapy this agent synergistically increased the effect of M12-PMO on this parameter in aged $m d x$ mice. This therapeutic combination significantly improved muscle endurance, unlike either agent when used as monotherapy, perhaps because correction of this functional deficit requires a threshold level of dystrophin expression (79). Dystrophin restoration by M12-PMO treatment was less pronounced in the present study than that reported in other preclinical studies, likely because of differences in dosing regimens $(52,80)$, but did closely resemble suboptimal dystrophin restoration observed clinically (13).

Synergistic effects on muscle function of therapeutic combinations incorporating $\operatorname{Smad} 2 / 3$ pathway inhibition have been observed previously in NMD models. Dumonceaux et al. found that shRNA-mediated knockdown of ActRIIB together with U7-DYS-mediated exon skipping produced greater maximal muscle force than either agent alone when injected into the TA muscle of young $m d x$ mice (81). Micro-dystrophin gene replacement in combination with follistatin delivery in aged $m d x$ mice also restored force generation and conferred resistance to contraction-induced injury (51). Combined use of exon skipping to restore dystrophin and inhibit GDF8 expression produced greater dystrophin expression than dystrophin exon skipping alone in both young and aged $m d x$ mice $(52,80)$. This treatment combination increased force generation in the young cohort (52) but was ineffective in the aged cohort (80), in contrast to the present study. In a mouse model of spinal muscular atrophy, follistatin (AAV1-follistatin) was administered in combination with a suboptimal dose of an SMN2 splicing modifier to successfully ameliorate muscle atrophy (82-84). These findings support our hypothesis that combination of gene modulation therapy with $S m a d 2 / 3$ inhibition by a multiligand trap such as ActRIIB:ALK4-Fc would augment the restoration of muscle function in patients with NMDs, particularly individuals with advanced disease.

In conclusion, the heterodimeric ligand trap ActRIIB:ALK4-Fc sequesters TGF- $\beta$ superfamily ligands with novel selectivity, increasing skeletal muscle mass and strength in mouse models of neuromuscular disorders while alleviating multiple comorbidities. Our findings provide further evidence to support the use of TGF- $\beta$ superfamily-based therapies in the treatment of DMD and other neuromuscular diseases, either as monotherapy or in combination with gene modulation therapies.

\section{Methods}

Construction, expression, and purification of recombinant ActRIIB:ALK4-Fc. ActRIIB:ALK4-Fc was generated by ligation of the extracellular domains (ECDs) of human ActRIIB (aa 19-134) and human ALK4 (aa 24-126) into separate vectors upstream of a modified human IgG1 Fc domain. The ECDs were generated by PCR using fulllength ActRIIB or ALK4 cDNA obtained from Invitrogen and Open Biosystems/Thermo Fisher Scientific, respectively, as templates. A modified human IgG1 Fc domain was obtained by PCR reactions using human IgG1 as a template (Invitrogen) and primers that introduced mutations designed to minimize homodimer formation. The ActRIIB ECD fragment was subcloned into the pAID4T vector containing the modified human IgG1 Fc domain. A second modified human IgG1 Fc with a different set of mutations was PCR-generated with a 6 xHis tag on the C-terminus, and the ALK4 ECD was subcloned into a separate pAID4T vector containing this modified human IgG1 Fc domain.

The 2 plasmids were coexpressed in Chinese hamster ovary (CHO) DUKX cells as a stable pool, resulting in the soluble expression of the fusion protein ActRIIB:ALK4-Fc. This was followed by dilution cloning for higher expression and then purification following a 4-step procedure. First, the protein was captured on a protein A MabSelect SuRe chromatography column (GE Healthcare), then washed and eluted with glycine at low $\mathrm{pH}$. Fractions from the elution were neutralized and pooled before being loaded over a Ni Sepharose 6 Fast flow column (GE Healthcare) and then eluted with an imidazole gradient. Eluted protein was further purified over an ActRIIB affinity column to ensure removal of impurities. Lastly, the protein was concentrated over a Q Sepharose column (GE Healthcare). The final material was dialyzed in PBS, and purity was assessed to be greater than $90 \%$ by SDS-PAGE gel with SimplyBlue SafeStain (Thermo Fisher Scientific) and analytical SEC column (Zenix-C SEC-300, Sepax). Another version of ActRIIB:ALK4-Fc, containing different mutations in the human IgG1, was also used in some assays and purified similarly, except in this case the Ni Sepharose step was replaced by a mixedmode CaptoBlue resin (GE Healthcare).

Antibodies and other reagents. ActRIIB-Fc and ALK1-Fc were produced internally as previously described $(23,26)$. Dual anti-GDF8/ anti-GDF11 antibody (RK35) (85), anti-activin A antibody (A2) (86), 
anti-GDF8 antibody (RK22) (30), and dual anti-ActRIIA/IIB antibody (BYM-338) (32) were modified internally for use in mice by substitution of murine IgG2a Fc. Antisense oligonucleotide M12-PMO (53) was synthesized by Gene Tools LLC.

Characterization of ligand binding. Ligand binding affinities for ActRIIB:ALK4-Fc and ActRIIB-Fc were assessed by SPR using a Biacore T200 instrument (GE Healthcare). All ligands used in the SPR study were produced in-house. In brief, goat anti-human Fc-specific IgG (Sigma-Aldrich) was immobilized on a Series S CM5 chip. A concentration series in 2-fold dilutions of activin A (0.01-2.5 nM), activin B (0.01-2.5 $\mathrm{nM})$, BMP9 (0.02-20 nM for ActRIIB:ALK4-Fc and 0.04-5 nM for ActRIIB-Fc), and GDF8 and GDF11 (0.04-20 nM) were injected in duplicate over experimental (captured ActRIIB:ALK4-Fc or ActRIIB-Fc) and control flow cells at a flow rate of $50 \mu \mathrm{L} / \mathrm{min}$ at $37^{\circ} \mathrm{C}$. Complex association and dissociation were monitored over 5 and 10 minutes, respectively. Surface regeneration between binding cycles was performed with 10 $\mathrm{mM}$ glycine, $\mathrm{pH}$ 1.7. The running buffer used for binding experiments contained 0.1 M HEPES, pH 7.4, 0.5 M NaCl, 3 mM EDTA, 0.005\% vol/ vol surfactant P2O, and $0.5 \mathrm{mg} / \mathrm{mL}$ BSA. Kinetic rate constants $k_{d}$ and $k_{a}$ and equilibrium dissociation constant $K_{D}$ were determined from double-referenced sensorgrams processed with BIAevaluation Software 3.1 (GE Healthcare) using a 1:1 interaction model with a mass transport term. Data shown in Table 1 are means \pm SEM calculated from multiple data sets obtained with various protein lots.

Mouse handling and sample collection. All experimental procedures, with the exception of the retinal studies (see below), were performed according to protocols approved by the Acceleron Pharma Institutional Animal Care and Use Committee. All mice in this study were male and were housed in standard cages under 12-hour light/12-hour dark cycles and fed ad libitum with a standard chow diet. Mice received vehicle (PBS) or ActRIIB:ALK4-Fc $(10 \mathrm{mg} / \mathrm{kg})$ s.c. unless otherwise indicated. Body weight and overall animal health were assessed during the dosing period, along with muscle force. Body composition was analyzed by nuclear magnetic resonance (NMR; Bruker Minispec). On completion of the studies, mice were euthanized by $\mathrm{CO}_{2}$ asphyxiation, and then individual muscle groups were removed and weighed. Tissues kept for histologic analysis were either frozen using 2-methylbutane cooled with liquid nitrogen, or fixed in $4 \%$ paraformaldehyde (Boston BioProducts). Deviations from these general methods are outlined below.

Animal models. Three major disease models were used in the present study: disuse atrophy, DMD, and ALS. Individual disease models are detailed below. In brief, 12-week-old WT C57BL/6J mice were used for the normal mouse, disuse atrophy, and in vivo protein synthesis studies. Both young (5-week-old) and aged (13- or 14-month-old) C57BL/10ScSn-Dmd ${ }^{m d x} / \mathrm{J}$ (BL10.mdx) mice were used for the DMD studies, with age-matched C57BL/10ScSn WT mice as the control. Seven-month-old DBA/2J- $m d x$ (D2.mdx) mice, along with age-matched control DBA/2J mice, were used for the respiratory studies. Five-weekold B6SJL-Tg (SOD1 ${ }^{\star}$ G93A)1Gur/J (SOD1 $\left.{ }^{\mathrm{G} 93 A}\right)$ mice were used for the ALS studies. All animals were obtained from The Jackson Laboratory.

Hind-limb unloading model. Mice were anesthetized under isoflurane, and the left hind limb was immobilized by application of a surgical staple to fix the ventral part of the foot and the distal part of the calf. The right hind limb was allowed to move freely, and served as the contralateral control. Treatment with ActRIIB:ALK4-Fc or vehicle was started at the time of staple installation, twice a week for 2 weeks, after which the mice were sacrificed (immobilized group). A second group was immobilized for 2 weeks without treatment, then remobilized (via removal of the staple under anesthesia), and subsequently treated with ActRIIB:ALK4-Fc or vehicle for another 2 weeks (remobilized group).

Micromechanical muscle injury model/acute fibrosis study. Fiveweek-old BL10.mdx mice were anesthetized with isoflurane, and the hind-limb skin was shaved and then disinfected with 70\% ethanol. Using micropins $(150 \mu \mathrm{m}$ diameter, fine headless pins; Kabourek, Czechoslovakia), 40 micropunctures were made daily in both TA muscles for 2 weeks; these were performed randomly over the whole surface, through the skin and deep into the muscle $(7-8 \mathrm{~mm})$, perpendicular to the longitudinal axis. The micropins induced several local myofiber injuries that, repeated daily, triggered acute fibrosis.

Respiratory studies. Mice were examined by unrestrained wholebody plethysmography (EMMS Supporting Science). All mice first received once-weekly training in chambers for 3 months. At a consistent time each week, mice remained in chambers for 40 minutes under normoxic conditions. After acclimation for 3 months, mice received pharmacologic treatments for 4 weeks. At study termination, mice were given 20 minutes to acclimate. Tidal volume was recorded over a 20-minute interval under normoxic conditions and then during acute hypoxia $\left(\mathrm{FiO}_{2}=0.1,20\right.$ minutes $)$.

Retinal studies. Retinal experiments were carried out in the laboratory of Paul Oh. The C57BL/6 WT mice for these studies were obtained from The Jackson Laboratory. Treatments were carried out on postnatal day 2 (P2) pups; the animals were treated with either saline or $2 \mathrm{mg} /$ $\mathrm{kg}$ of either ALK1-Fc, ActRIIB-Fc, or ActRIIB:ALK4-Fc. Animals were sacrificed on $\mathrm{P} 8$, and then retinas were prepared for staining.

Muscle contractility. Forelimb grip strength was examined in WT mice according to the TREAT-NMD protocol (https://treat-nmd.org/ sop/dmd_m-2-2-001/). Measurements of isometric and eccentric contractions by the TA muscle in situ and ankle dorsiflexion in vivo were performed as previously described (87). Measurement of isometric contraction by diaphragm muscle ex vivo was conducted as previously described (88). Testing was carried out in Ringer's solution maintained at $22^{\circ} \mathrm{C}$. Optimal muscle length and twitch force were recorded under a single stimulus using a 200-microsecond square pulse. Tetanic force-frequency relationship was conducted with stimulation by square wave stimuli of 200 milliseconds. Absolute values of tension were normalized to the physiological cross-sectional area.

Electrophysiology. Electrophysiologic measurements were performed on the left hind limb using Synergy (Natus Medical Incorporated). Briefly, the sciatic nerve was supramaximally stimulated at the sciatic notch, and the entire distal leg muscle compartment recorded via disposable ring electrodes, with a ground electrode placed on the right hind paw to record the CMAP amplitude. Low-frequency $(3 \mathrm{~Hz})$ repetitive nerve stimulations were performed on the sciatic nerve, and recorded on the entire distal leg muscle compartment. The fractional decrease in amplitude of the CMAP was calculated using the amplitude (peak positive to peak negative) of the first and fourth responses.

Immunoblotting. Briefly, TA muscles were homogenized in RIPA buffer with protease inhibitors (1:100). Proteins were separated on 10\% SDS-PAGE acrylamide gels, transferred to PVDF membranes, and stained with Ponceau S. Puromycin-labeled proteins were detected with a mouse IgG2a monoclonal anti-puromycin antibody (1:5000; Developmental Studies Hybridoma Bank [DSHB]), followed by incubation with an HRP-conjugated anti-mouse IgG2a secondary 
antibody (1:50,000; Jackson ImmunoResearch Laboratories Inc.). Enhanced chemiluminescence (ECL) reagent (Cell Signaling Technology) was used for detection. Blots were stripped and reprobed with a GAPDH antibody (Cell Signaling Technology). Dystrophin expression was detected as previously described (52). Densitometric analysis was performed using Image J software (NIH).

TaqMan real-time PCR. Total RNA was extracted from muscle lysates using the Ambion RiboPure kit (Thermo Fisher Scientific). RNA (500 ng) was reverse-transcribed with iScript cDNA synthesis kit (Bio-Rad). The cDNA generated was used to perform real-time PCR to determine mRNA levels for Agrin, Musk, Lrp4, Dok7, Rapsyn, and $A c t b$ (primers from Thermo Fisher Scientific), with amplifications performed using a 7300 Real-Time PCR System (Applied Biosystems).

MicroRNA analysis. MicroRNA isolation from mouse serum was performed using a MagMAX mirVana kit (Thermo Fisher Scientific) according to the manufacturer's instructions. Reverse transcription (RT) was then carried out using the TaqMan MicroRNA Reverse Transcription Kit (Thermo Fisher Scientific). PCR primer pools for miR-206 (or miR146a, miR-1, miR-21, miR-133b), miR-16, and cel-miR-39 were combined with the RT product to make up a $25 \mu \mathrm{L}$ final reaction mix. The preamplification reaction was carried out for 14 cycles on a MiniAmp Thermal Cycler (Applied Biosystems). Quantitative RT-PCR was performed using TaqMan Fast Advanced Master Mix with preamplification product on a QuantStudio 6 Flex system (Thermo Fisher Scientific). Relative expression levels were analyzed using DataAssist software (Applied Biosyste$\mathrm{ms}$ ) and normalized to miR-16 and cel-miR-39.

Histology and immunofluorescence. Fixed C2C12 cells, cryosectioned TA muscle, and eye as well as teased-out single myofibers were stained, following standard immunofluorescent protocol. The primary antibodies used were a phospho-specific Smad3 antibody (Abcam) (87), anti-MHCI (BA-F8, DSHB), anti-MHCIIa (SC-71, DSHB), anti-MHCIIx (6H1, DSHB), anti-MHCIIb (BF-F3, DSHB), anti-MHCIIx (BF-35, DSHB), an isolectin B4 (IB4) biotin conjugate (MilliporeSigma), an anti-neurofilament antibody (Millipore), and an anti-synaptophysin antibody (Invitrogen); and AChRs were labeled with $\alpha$-bungarotoxin (Invitrogen).

Histology and immunohistochemistry. Sections of paraffin-embedded TA muscle were stained with Picrosirius red (0.1\% in saturated picric acid; Rowley Biochemical Inc.) to determine muscle fibrosis or were incubated with a rabbit anti-dystrophin antibody (1:2000; Abcam), followed by a polymer secondary antibody to identify dystrophin.

Quantification of histology. Area of myocytes was assessed as previously described (53). In brief, sections were visualized with a Zeiss LSM confocal microscope, and tile scans were performed to assemble the entire cross-section image. At least 100 muscle fibers were assessed, except for the ones in DMD studies, in which the entire cross-section area was counted. For dystrophin quantification, all fibers within the entire cross section were counted and assessed. Only fibers showing continuous staining of dystrophin were considered as dystrophin positive and evaluated as a percentage of the number of total fibers of the entire section. For fibrosis quantification, an intensity threshold was set up for Picrosirius red (fibrosis) channel for all sections. Only the area above the threshold was counted as fibrotic. The positive fibrotic area was normalized to the entire section. To determine NMJ morphology, NMJs were imaged at $2 \mu \mathrm{m}$ intervals using $\times 40$ oil-immersion objectives on a Zeiss LSM confocal microscope and merged using maximal projections of $Z$-stacks. Approximately 10-15 AChR staining areas per sample were quantified using Image $(\mathrm{NIH})$. AChR islet number was determined as previously described (70). Endplates (AChR clusters) were classified as fully occupied, partially occupied, or unoccupied by nerve terminals and expressed as the percentage of total endplates.

Statistics. All values are expressed as means \pm SEM. Significance was determined by either unpaired Student's $t$ tests or 1-way ANOVA followed by either Tukey's or Dunnett's method. All tests were 2-tailed. $P$ values less than or equal to 0.05 were considered statistically significant. GraphPad Prism version 7.03 (GraphPad Software) was used for statistical analysis.

Study approval. All housing, breeding, and procedures were performed according to the NIH Guide for the Care and Use of Laboratory Animals (National Academies Press, 2011) and approved by Dignity Health/ Barrow Neurological Institute Animal Care and Use Committee.

\section{Author contributions}

RSP, JL, RNVSS, RC, AVG, and RK designed the study. JL, MF, MC, TB, PAM, KL, DS, PO, KW, EPB, MCM, RG, LK, and HC carried out the experiments. JL, RSP, DS, RC, AVG, RNVSS, PO, SK, and RK analyzed the data. JL, RNVSS, SK, and MJA wrote the manuscript, which was reviewed by all authors.

\section{Acknowledgments}

The authors thank the Protein Science group at Acceleron Pharma for generation of ActRIIB:ALK4-Fc and other constructs used in this study. A special thanks to Chien-Ping Ko and Zhihua Feng at University of Southern California for help with the NMJ staining.

Address correspondence to: Rajasekhar N.V.S. Suragani, Acceleron Pharma Inc., 128 Sidney Street, Cambridge, MA 02139 USA. Phone: 617.649.9343; Email: rsuragani@xlrn.com.

AVG's and KL's present address is: Dragonfly Therapeutics, Waltham, Massachusetts, USA.

PAM's present address is: Simcere Innovation Inc., Cambridge, Massachusetts, USA.
1. Rudrappa SS, et al. Human skeletal muscle disuse atrophy: effects on muscle protein synthesis, breakdown, and insulin resistance - a qualitative review. Front Physiol. 2016;7:361.

2. Yoshida T, Delafontaine P. Mechanisms of cachexia in chronic disease states. Am JMed Sci. 2015;350(4):250-256.

3. Morrison BM. Neuromuscular diseases. Semin Neurol. 2016;36(5):409-418.

4. Andrews JG, Wahl RA. Duchenne and Becker muscular dystrophy in adolescents: current perspectives. Adolesc Health Med Ther . 2018;9:53-63.

5. Bladen CL, et al. The TREAT-NMD DMD Global Database: analysis of more than 7,000 Duchenne muscular dystrophy mutations. Hum Mutat. 2015;36(4):395-402.

6. Ervasti JM, et al. Deficiency of a glycoprotein component of the dystrophin complex in dystrophic muscle. Nature. 1990;345(6273):315-319.

7. Mann CJ, et al. Aberrant repair and fibrosis development in skeletal muscle. Skelet Muscle. 2011;1(1):21.

8. Ishikawa Y, et al. Duchenne muscular dystrophy: survival by cardio-respiratory interventions. Neuromuscul Disord. 2011;21(1):47-51

9. Gloss D, et al. Practice guideline update summary: corticosteroid treatment of Duchenne muscular dystrophy: report of the Guideline Development Subcommittee of the American Academy of Neurology. Neurology. 2016;86(5):465-472. 
10. Rodino-Klapac LR, et al. Gene therapy for Duchenne muscular dystrophy: expectations and challenges. Arch Neurol. 2007;64(9):1236-1241.

11. Rodino-Klapac LR. MicroRNA based treatment of cardiomyopathy: not all dystrophies are created equal. JAm Heart Assoc. 2013;2(4):e000384.

12. Benedetti S, et al. Repair or replace? Exploiting novel gene and cell therapy strategies for muscular dystrophies. FEBS J. 2013;280(17):4263-4280.

13. Charleston JS, et al. Eteplirsen treatment for Duchenne muscular dystrophy: exon skipping and dystrophin production. Neurology. 2018;90(24):e2146-e2154.

14. Gordon KJ, Blobe GC. Role of transforming growth factor- $\beta$ superfamily signaling pathways in human disease. Biochim Biophys Acta. 2008;1782(4):197-228.

15. McPherron AC, Lee SJ. Double muscling in cattle due to mutations in the myostatin gene. Proc Natl Acad Sci U S A. 1997;94(23):12457-12461.

16. Lee SJ, et al. Regulation of muscle mass by follistatin and activins. Mol Endocrinol. 2010;24(10):1998-2008.

17. Mariot V, et al. Downregulation of myostatin pathway in neuromuscular diseases may explain challenges of anti-myostatin therapeutic approaches. Nat Commun. 2017;8(1):1859.

18. Chen JL, et al. Specific targeting of TGF- $\beta$ family ligands demonstrates distinct roles in the regulation of muscle mass in health and disease. Proc Natl Acad Sci U S A. 2017;114(26):E5266-E5275.

19. Latres E, et al. Activin A more prominently regulates muscle mass in primates than does GDF8. Nat Commun. 2017;8:15153.

20. Sartori R, et al. Smad 2 and 3 transcription factors control muscle mass in adulthood. Am JPhysiol Cell Physiol. 2009;296(6):C1248-C1257.

21. Aykul S, Martinez-Hackert E. Transforming growth factor- $\beta$ family ligands can function as antagonists by competing for type II receptor binding. J Biol Chem. 2016;291(20):10792-10804.

22. Campbell C, et al. Myostatin inhibitor ACE-031 treatment of ambulatory boys with Duchenne muscular dystrophy: results of a randomized, placebo-controlled clinical trial. Muscle Nerve. 2017;55(4):458-464.

23. Sako D, et al. Characterization of the ligand binding functionality of the extracellular domain of activin receptor type IIb. J Biol Chem. 2010;285(27):21037-21048.

24. Townson SA, et al. Specificity and structure of a high affinity activin receptor-like kinase 1 (ALK1) signaling complex. J Biol Chem. 2012;287(33):27313-27325.

25. Ntumba K, et al. BMP9/ALK1 inhibits neovascularization in mouse models of age-related macular degeneration. Oncotarget. 2016;7(35):55957-55969.

26. Mitchell D, et al. ALK1-Fc inhibits multiple mediators of angiogenesis and suppresses tumor growth. Mol Cancer Ther. 2010;9(2):379-388.

27. Pistilli EE, et al. Targeting the activin type IIB receptor to improve muscle mass and function in the mdx mouse model of Duchenne muscular dystrophy. Am J Pathol. 2011;178(3):1287-1297.

28. Morey-Holton ER, Globus RK. Hindlimb unloading rodent model: technical aspects. JAppl Physiol (1985). 2002;92(4):1367-1377.

29. Brooks NE, Myburgh KH. Skeletal muscle wast- ing with disuse atrophy is multi-dimensional: the response and interaction of myonuclei, satellite cells and signaling pathways. Front Physiol. 2014;5:99.

30. Wagner KR, et al. A phase I/II trial of MYO-O29 in adult subjects with muscular dystrophy. Ann Neurol. 2008;63(5):561-571.

31. St Andre M, et al. A mouse anti-myostatin antibody increases muscle mass and improves muscle strength and contractility in the mdx mouse model of Duchenne muscular dystrophy and its humanized equivalent, domagrozumab (PF06252616), increases muscle volume in cynomolgus monkeys. Skelet Muscle. 2017;7(1):25.

32. Morvan F, et al. Blockade of activin type II receptors with a dual anti-ActRIIA/IIB antibody is critical to promote maximal skeletal muscle hypertrophy. Proc Natl Acad Sci US A. 2017;114(47):12448-12453.

33. Harish $\mathrm{P}$, et al. Inhibition of myostatin improves muscle atrophy in oculopharyngeal muscular dystrophy (OPMD). JCachexia Sarcopenia Muscle. 2019;10(5):1016-1026.

34. McGreevy JW, et al. Animal models of Duchenne muscular dystrophy: from basic mechanisms to gene therapy. Dis Model Mech. 2015;8(3):195-213.

35. Hakim CH, et al. The passive mechanical properties of the extensor digitorum longus muscle are compromised in 2- to 20-mo-old mdx mice. $J$ Appl Physiol (1985). 2011;110(6):1656-1663.

36. Zaharieva IT, et al. Dystromirs as serum biomarkers for monitoring the disease severity in Duchenne muscular dystrophy. PLoS One. 2013;8(11):e80263.

37. Mizuno $\mathrm{H}$, et al. Identification of muscle-specific microRNAs in serum of muscular dystrophy anima models: promising novel blood-based markers for muscular dystrophy. PLoS One. 2011;6(3):e18388.

38. Fiorillo AA, et al. TNF- $\alpha$-induced microRNAs control dystrophin expression in Becker muscular dystrophy. Cell Rep. 2015;12(10):1678-1690.

39. Klingler W, et al. The role of fibrosis in Duchenne muscular dystrophy. Acta Myol. 2012;31(3):184-195.

40. Desguerre I, et al. A new model of experimental fibrosis in hindlimb skeletal muscle of adult mdx mouse mimicking muscular dystrophy. Muscle Nerve. 2012;45(6):803-814.

41. Huang $P$, et al. Impaired respiratory function in $\mathrm{mdx}$ and $\mathrm{mdx} / \mathrm{utrn}^{(+/-)}$mice. Muscle Nerve. 2011;43(2):263-267.

42. Coley WD, et al. Effect of genetic background on the dystrophic phenotype in mdx mice. Hum Mol Genet. 2016;25(1):130-145.

43. Pratt SJP, et al. Pre- and postsynaptic changes in the neuromuscular junction in dystrophic mice. Front Physiol. 2015;6:252.

44. Theroux MC, et al. C Histomorphology of neuromuscular junction in Duchenne muscular dystrophy. Pediatr Anesth. 2008;18(3):256-259.

45. van der Pijl EM, et al. Characterization of neuromuscular synapse function abnormalities in multiple Duchenne muscular dystrophy mouse models. Eur J Neurosci. 2016;43(12):1623-1635.

46. Li L, et al. Neuromuscular junction formation, aging, and disorders. Annu Rev Physiol. 2018;80:159-188.

47. Lyons PR, Slater CR. Structure and function of the neuromuscular junction in young adult $\mathrm{mdx}$ mice. J Neurocytol. 1991;20(12):969-981.

48. Pratt SJP, et al. Recovery of altered neuromuscular junction morphology and muscle function in mdx mice after injury. Cell Mol Life Sci. 2015;72(1):153-164.

49. Burden SJ, et al. Fundamental molecules and mechanisms for forming and maintaining neuromuscular synapses. Int J Mol Sci. 2018;19(2):490.

50. Aartsma-Rus A. Overview on DMD exon skipping. Methods Mol Biol. 2012;867:97-116.

51. Rodino-Klapac LR, et al. Micro-dystrophin and follistatin co-delivery restores muscle function in aged DMD model. Hum Mol Genet. 2013;22(24):4929-4937.

52. Lu-Nguyen $\mathrm{N}$, et al. Systemic antisense therapeutics for dystrophin and myostatin exon splice modulation improve muscle pathology of adult mdx mice. Mol Ther Nucleic Acids. 2017;6:15-28.

53. Gao X, et al. Effective dystrophin restoration by a novel muscle-homing peptide-morpholino conjugate in dystrophin-deficient mdx mice. Mol Ther. 2014;22(7):1333-1341.

54. Mary P, et al. Neuromuscular diseases: diagnosis and management. Orthop Traumatol Surg Res. 2018;104(1S):S89-S95.

55. Lee SJ, et al. Regulation of muscle growth by multiple ligands signaling through activin type II receptors. Proc Natl Acad Sci U S A. 2005;102(50):18117-18122.

56. Sartori R, et al. BMP signaling controls muscle mass. Nat Genet. 2013;45(11):1309-1318.

57. Newman AB, et al. Strength and muscle quality in a well-functioning cohort of older adults: the Health, Aging and Body Composition Study. J Am Geriatr Soc. 2003;51(3):323-330.

58. Stedman HH, et al. The mdx mouse diaphragm reproduces the degenerative changes of Duchenne muscular dystrophy. Nature. 1991;352(6335):536-539.

59. Bo Li Z, et al. Inhibition of myostatin reverses muscle fibrosis through apoptosis. J Cell Sci. 2012;125(Pt 17):3957-3965.

60. Zhu J, et al. Follistatin improves skeletal muscle healing after injury and disease through an interaction with muscle regeneration, angiogenesis, and fibrosis. Am J Pathol. 2011;179(2):915-930.

61. Bo Li Z, et al. Myostatin directly regulates skeletal muscle fibrosis. J Biol Chem. 2008;283(28):19371-19378.

62. de Kretser DM, et al. The roles of activin A and its binding protein, follistatin, in inflammation and tissue repair. Mol Cell Endocrinol. 2012;359(1-2):101-106.

63. Yaden BC, et al. Inhibition of activin A ameliorates skeletal muscle injury and rescues contractile properties by inducing efficient remodeling in female mice. Am J Pathol. 2014;184(4):1152-1166.

64. Pessina P, et al. Novel and optimized strategies for inducing fibrosis in vivo: focus on Duchenne muscular dystrophy. Skelet Muscle. 2014;4:7.

65. Katayama K, et al. Hypoxia exaggerates inspiratory accessory muscle deoxygenation during hyperpnoea. Respir Physiol Neurobiol. 2015;211:1-8.

66. Slater CR. The structure of human neuromuscular junctions: some unanswered molecular questions. Int J Mol Sci. 2017;18(10):2183.

67. Singhal N, Martin PT. Role of extracellular matrix proteins and their receptors in the development 


\section{RESEARCH ARTICLE}

of the vertebrate neuromuscular junction. Dev Neurobiol. 2011;71(11):982-1005.

68. Rudolf R, et al. Degeneration of neuromuscular junction in age and dystrophy. Front Aging Neurosci. 2014;6:99.

69. Fish LA, Fallon JR. Multiple MuSK signaling pathways and the aging neuromuscular junction. Neurosci Lett. 2020;731:135014.

70. Pratt SJP, et al. Effects of in vivo injury on the neuromuscular junction in healthy and dystrophic muscles. J Physiol. 2013;591(2):559-570.

71. Ibebunjo C, et al. Genomic and proteomic profiling reveals reduced mitochondrial function and disruption of the neuromuscular junction driving rat sarcopenia. Mol Cell Biol. 2013;33(2):194-212.

72. McCabe BD, et al. The BMP homolog Gbb provides a retrograde signal that regulates synaptic growth at the Drosophila neuromuscular junction. Neuron. 2003;39(2):241-254.

73. Baines RA. Synaptic strengthening mediated by bone morphogenetic protein-dependent retrograde signaling in the Drosophila CNS. J Neurosci. 2004;24(31):6904-6911.

74. Berke B, et al. Target-dependent retrograde signaling mediates synaptic plasticity at the Drosophila neuromuscular junction. Dev Neurobiol.
2019;79(11):895-912.

75. Augustin H, et al. Myostatin-like proteins regulate synaptic function and neuronal morphology. Development. 2017;144(13):2445-2455.

76. Gratacos E, et al. Bone morphogenetic protein- 6 is a neurotrophic factor for calbindin-positive striatal neurons. JNeurosci Res. 2002;70(5):638-644.

77. Galter D, et al. Differential regulation of distinct phenotypic features of serotonergic neurons by bone morphogenetic proteins. Eur J Neurosci. 1999;11(7):2444-2452.

78. Cordova G, et al. Combined therapies for Duchenne muscular dystrophy to optimize treatment efficacy. Front Genet. 2018;9:114.

79. van der Pijl EM, et al. Low dystrophin levels are insufficient to normalize the neuromuscular synaptic abnormalities of mdx mice. Neuromuscul Disord. 2018;28(5):427-442.

80. Lu-Nguyen N, et al. Functional muscle recovery following dystrophin and myostatin exon splice modulation in aged mdx mice. Hum Mol Genet. 2019;28(18):3091-3100.

81. Dumonceaux J, et al. Combination of myostatin pathway interference and dystrophin rescue enhances tetanic and specific force in dystrophic mdx mice. Mol Ther. 2010;18(5):881-887.
82. Feng Z, et al. Pharmacologically induced mouse model of adult spinal muscular atrophy to evaluate effectiveness of therapeutics after disease onset. Hum Mol Genet. 2016;25(5):964-975.

83. Rose FF Jr, et al. Delivery of recombinant follistatin lessens disease severity in a mouse model of spinal muscular atrophy. Hum Mol Genet. 2009;18(6):997-1005.

84. Bowerman M, et al. Therapeutic strategies for spinal muscular atrophy: $\mathrm{SMN}$ and beyond. Dis Model Mech. 2017;10(8):943-954.

85. Apgar JR, et al. Beyond CDR-grafting: structure-guided humanization of framework and CDR regions of an anti-myostatin antibody. MAbs. 2016;8(7):1302-1318.

86. Zhou X, et al. Reversal of cancer cachexia and muscle wasting by ActRIIB antagonism leads to prolonged survival. Cell. 2010;142(4):531-543.

87. Pearsall RS, et al. Follistatin-based ligand trap ACE-083 induces localized hypertrophy of skeletal muscle with functional improvement in models of neuromuscular disease. Sci Rep. 2019;9(1):11392.

88. Percival JM, et al. Sildenafil reduces respiratory muscle weakness and fibrosis in the $\mathrm{mdx}$ mouse model of Duchenne muscular dystrophy. J Pathol. 2012;228(1):77-87. 